\title{
ON THE STRUCTURE OF HIGH-ENERGY TWO-BODY NON-DIFFRACTIVE REACTIONS*
}

\author{
M. ROSS, F. S. HENYEY and G. L. KANE \\ Physics Department, University of Michigan, \\ Ann Arbor, Michigan 48104
}

Received 12 June 1970

\begin{abstract}
We consider the structure of two-body scattering amplitudes, concentrating on those reactions in which the "internal" quantum numbers exchanged correspond to the quantum numbers of a single particle, different from those of the vacuum. We compare conventional Regge-pole models (in which dips as a function of $t$ are caused by "nonsense" factors) with our strong-cut model (in which dips are caused by a diffractive mechanism). In the conventional models, the systematics of the angular structure are determined by the nature of the exchange, as well as by the helicity structure and factorization. In the strong-cut model, the systematics of the angular structure are determined exclusively by the helicity structure of the amplitude (except for slight modification in the case of pion exchange, due to the proximity of the pion pole to the physical region); most important is the net helicity flip of the amplitude. We examine experimental data, particularly with regard to dips near $-t$ (or $-u$ ) $\approx 0.2$ or 0.6 , and near $t=0$. It is found that whenever a distinction can be made, the strong-cut model is favored over the conventional models (even including cuts determined by conventional absorption models). Further experimental tes'ts are proposed. Double-particle exchange processes, energy dependence, effects of $t$-channel unitarity and duality are discussed.
\end{abstract}

\section{INTRODUCTION}

In this paper we discuss the energy dependence and the angular dependence of particle exchange high-energy two-body processes. These are all processes that cannot proceed by the exchange of a system with the quantum numbers of the vacuum. We are particularly interested in separating effects which arise from details of the exchange mechanism from those which are due to the structure of absorptive effects.

The structure we are primarily concerned with is the dip and bump structure seen in the angular distribution of some cross sections, and the related "crossover" zeros seen in the difference of elastic cross sections.

In the conventional Regge-pole models used to describe the reactions we are considering, these effects are attributed to various causes: accidental vanishings of the Regge-pole form factors (i.e. factorized residues), socalled "nonsense" zeros, and effects of exchange degeneracy.

\footnotetext{
* Research supported in part by the US Atomic Energy Commission.
} 
However in the absorption model, these effects can be attributed to one single effect, related to similar structure in many branches of physics other than high-energy physics. In the absorption model a reaction has two aspects, exchange and shadowing. The shadowing of the rear part of each particle by its front part during the collision decreases the likelihood that a particular exchange will occur in a head-on collision of the two objects. If the shadowing is strengthened compared to estimates based on elastic scattering it results in strong suppression of the reaction in a head-on collision so that the exchange can occur only if the particles pass at a certain distance from each other. If they pass at a greater or lesser distance, there is little possibility of exchange. Thus, there is a relatively sharp boundary in impact parameter at which the process occurs. This is similar to light passing a small black object yielding a diffraction pattern. The rather obvious analogy to be drawn from similar patterns at high-energy has been rejected up to now because a sharp boundary is known not to be present in the matter distributions of strongly interacting particles. Using electrons as probes they have been found to be fuzzy objects. The strong absorption theory takes the fuzziness into account and yet predicts the sharp boundary for exchange reactions.

It is well established that virtual hadronic particles are composite, and therefore must be Reggeized. One can easily see that absorption, which is related to the geometrical structure of the scattering particles, has no ef fect on the angular momentum properties of an elementary exchanged particle. Thus, it is essential to have a Regge pole input to the absorption model.

The two possible sources of the angular structure are that the structure be part of the exchange mechanism, as is typical of conventional Reggepole amplitudes, slightly modified perhaps by absorption effects, or that it be due to strong absorption effects superimposed on a smooth Regge pole amplitude. The latter theory [1] is called "strong-cut Reggeized absorption model" or SCRAM. In its present form SCRAM contains undetermined but restricted parameters which are always constrained to yield the appropriate diffraction patterns.

These two mechanisms for patterns in the angular distributions can be distinguished by examining the helicity dependence of the angular structure. In many cases displaced patterns from amplitudes with different helicities are on top of each other and must be disentangled. By examining certain crucial experiments where a small number of helicity amplitudes dominate, the two mechanisms can be distinguished, and the strong-absorption model is found to be correct.

The significance of this success is twofold: it establishes a correct description of simple processes which can be used as a check for theories with wider application. It demonstrates that very simple mathematical behavior does not describe high-energy reactions in detail; instead, physical models like those of optics and low-energy nuclear physics apply. Thus we establish that Regge pole exchange occurs in the amplitude as a "Born approximation", and we establish its form; on the other hand, the amplitude does not consist solely of Regge pole exchange, and the obvious angular structure is due to the other effects. 


\section{EXCHANGE ANALYSIS OF $2 \rightarrow 2$ REACTIONS ${ }^{*}$}

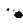

\subsection{General features}

High-energy two-body reactions are observed to have angular distributions peaked at the forward and backward directions. At high enough energy, the distinction between the forward scattering cross section and the backward scattering cross section can be made unambiguously. When we write the reaction as $a+b \rightarrow c+d$ we refer to the forward cross section, i.e., that part in which $c$ continues in approximately the same direction as a. (The remainder of the cross section is then written $a+b \rightarrow d+c$.) We observe this convention throughout this paper.

A very important characterization of the reaction $a+b \rightarrow c+d$ is the internal quantum numbers, $B, Y, I, Q$, etc., (but not $J, P$ ) lost by a in forming c, i.e., the internal quantum numbers of the $\bar{c}+$ a system (see fig. 1). The observed size of the cross section and its energy dependence depend on whether these quantum numbers are identical to the quantum numbers of the vacuum, of one particle, or of two, as spelled out in table 1.

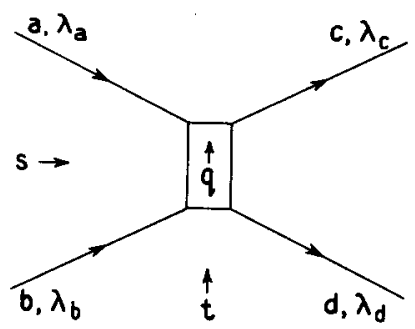

Fig. 1. Kinematics of the reaction $a+b \rightarrow c+d$. Each particle has helicity $\lambda_{j}$.

The momentum transfer $q$, and the $s$ - and $t$-channels are shown.

These results demand some kind of particle exchange description of high-energy reactions, with hopefully rapid convergence in the number of particles exchanged. The energy dependence of type- $R$ reactions (see definition in table 1 ) does not agree with elementary particle exchange:

$$
\sigma \propto \frac{\mathrm{d} \sigma}{\mathrm{d} t} \propto s^{2 J-2},
$$

where $J$ is the spin of the exchanged particle. Instead an approximate dependence

$$
\frac{\mathrm{d} \sigma}{\mathrm{d} t} \propto s^{2 \alpha(t)-2},
$$

has been observed for a number of cases with the extrapolation of $\alpha(t)$ yielding the observed spin, of the exchanged particle i.e., $\alpha\left(m^{2}\right)=J$. The best example is $\pi^{-} p \rightarrow \pi^{0} n$ measured by Sonderegger and collaborators [3]. They find an approximately straight trajectory $\alpha(t)$ extrapolating through

\footnotetext{
* Much of the material in this section is well known, see e.g.the review by Barger [2].
} 
Table 1

Relationship between the cross section for $P_{1 \mathrm{ab}} \gtrsim 3 \mathrm{GeV}$ and the exchanged quantum numbers.

\begin{tabular}{|c|c|c|c|c|}
\hline Type & $\begin{array}{c}\text { Internal } \\
\text { quantum numbers } \\
\text { correspond to }\end{array}$ & $\begin{array}{l}\text { Example } \\
\text { of } \\
\text { reaction }\end{array}$ & $\begin{array}{l}\text { Relative size } \\
\text { of } \\
\text { cross section }\end{array}$ & $\begin{array}{l}\text { Energy dependence } \\
\text { of integrated } \\
\text { cross section }\end{array}$ \\
\hline V & vacuum & elastic & large & approx. constant \\
\hline $\mathbf{R}$ & one particle & $\begin{array}{c}\mathrm{pp} \rightarrow \mathrm{N}_{\frac{1}{2}}^{*} \mathrm{p} \\
\pi^{-} \mathrm{p} \rightarrow \pi^{\mathrm{O}} \mathrm{n} \\
\pi^{-} \mathrm{p} \rightarrow \mathrm{K}^{\mathrm{O}} \Sigma^{\mathrm{O}}\end{array}$ & $\begin{array}{c}\text { large at } \\
\text { low energy }\end{array}$ & $\begin{array}{c}s^{-n} \\
-1 \gtrsim n \gtrsim-4\end{array}$ \\
\hline $\mathbf{R}^{2}$ & two particles & $\pi^{-} \mathrm{p} \rightarrow \mathrm{K}^{+} \Sigma^{-}$ & very small & $?$ \\
\hline
\end{tabular}

unity at $t=m_{\rho}^{2}$. Other experiments have yielded $\alpha_{\pi} \approx 0$ for small $-t$ (réf. [4]), and $\alpha_{\mathrm{N}}(u), \alpha_{\Delta}(u)$ approximately linear trajectories extrapolating back roughly to $\alpha=\frac{1}{2}, \frac{3}{2}$ at $M_{\mathrm{N}}^{2}$ and $M_{\triangle}^{2}$, respectively [5-10].

These results suggest that virtual particles (e.g., those exchanged at high $s$ and with $t$ of order $M^{2}$ ) should be described as Reggeons. (Thus, instead of considering a single particle of fixed spin, the $t$-channel partial wave amplitude has a moving pole in the $j$-plane; the trajectory involves a series of particles or resonances at appropriate values of $j, t$ ). This result is very appealing since it corresponds to a composite picture for particles (e.g., bound and excited states in Schroedinger theory lie on Regge trajectories).

The V-type reactions (i.e. vacuum exchange; see table 1) receive contributions from Reggeon exchange (e.g., $f_{o}$ exchange). However, the main contribution, described by the term "diffraction" or Pomeron exchange, is probably not particle exchange. Its mathematical form is not known. We will not, then, discuss elastic or diffractive, inelastic processes. We hope, however, that when we fold diffractive effects with particle exchange processes as discussed below, that detailed knowledge of the diffractive effects will be unimportant.

Consider a reaction with Reggeon exchange allowed. The exchange of more than one Reggion is hopefully a relatively small correction (to be dis cussed immediately below). It is clear, however, that vacuum exchange effects should accompany the exchange of a Reggeon. The physical arguments are discussed in detail in HKPR [1]. Double Reggeon exchange and Reggeonvacuum exchange amplitudes are Regge cuts [11]. We will call vacuum exchange contributions associated with a particular Reggeon exchange the "principal cut" associated with that Regge pole. Another term we use is the "absorption correction".

The principal cuts or absorption corrections may be relatively large compared to the Regge pole. A main purpose of this paper is to present some evidence for large principal cuts. The calculation of principal cuts, based on ideas of absorption, is discussed in sect. 3 below. 


\subsection{Double-Reggeon exchange ( $\mathrm{R}^{2}$ type reactions)}

At present in the development of the theory of high-energy two-body reactions, one recognizes the possible importance of two-Reggeon exchange but is unable to find definitive experimental evidence or to do any kind of complete calculation. We limit consideration of two-Reggeon exchange reactions to this introductory section. The experimental evidence is of two kinds: (i) observation of processes requiring double-Reggeon (especially double charged meson) exchange, (ii) deviation of the ratio of two singleexchange "allowed" reactions from the value expected from the isotopic spin of the single exchange. At low energy $\left(P_{\mathrm{L}}<3 \mathrm{GeV} / c\right)$ the rates for doubleexchange processes are not small, but are falling very rapidly with energy $[12,13]$. (We note, in this connection, that several allowed (R-type) processes also fall much more rapidly as a function of $s$ at low energy than they do at high energy.) At higher energy the rates are very small. There is no reason (theoretical or experimental) to believe that the rates continue to drop very rapidly with energy.

The three reported observations are

$$
\begin{aligned}
\pi^{-} \mathrm{p} & \rightarrow \mathrm{K}^{+} \mathrm{Y}_{1}^{*}, \\
\mathrm{p} \overline{\mathrm{p}} & \rightarrow \Sigma^{-} \overline{\Sigma^{-}}, \\
\mathrm{K}^{-} \mathrm{p} & \rightarrow \pi^{+} \Sigma^{-} .
\end{aligned}
$$

The evidence for the first [14] $\dagger$ at the highest energy, $4.5 \mathrm{GeV} / c$, is poor, because of the possibility of reflections, e.g., confusion with the final state $\mathrm{K}^{*} \Lambda$ in (2). Reaction (3) (ref. [15]) is observed at $5.7 \mathrm{GeV} / c$. Its ratio to the type-R reaction $p \bar{p} \rightarrow \Sigma^{+} \Sigma^{+}$is found to be

$$
\frac{\sigma\left(\mathrm{p} \bar{p} \rightarrow \Sigma^{-} \overline{\Sigma^{-}}\right)}{\sigma\left(p \bar{p} \rightarrow \Sigma^{+}\right.}=\frac{1.3 \pm 0.4 \mu \mathrm{b}}{33 \pm 4 \mu \mathrm{b}},
$$

and it is observed, as expected theoretically, that the double exchange process has a flatter angular distribution than the single exchange. At 4.06 and $5.47 \mathrm{GeV} / c$, (combined experiments) reaction (4) (ref. [16]) has been observed.

$$
\frac{\sigma\left(K^{-} p \rightarrow \pi^{+} \Sigma^{-}\right)}{\sigma\left(K^{-} p \rightarrow \pi^{-} \Sigma^{+}\right)}=\frac{2 \text { events }}{395 \text { events }} \approx \frac{1}{200} .
$$

Upper limits have been determined in other reactions:

$$
\begin{aligned}
& \pi^{-} \mathrm{p} \rightarrow \mathrm{K}^{+} \Sigma^{-}, \\
& \mathrm{K}^{-} \mathrm{p} \rightarrow \mathrm{pK}^{-} .
\end{aligned}
$$

One finds in the 4-5 GeV range [17]

$\dagger$ We would like to thank . Exack for sending us Dalitz plots of the data. 


$$
\frac{\sigma\left(\pi^{-} p \rightarrow K^{+} \Sigma^{-}\right)}{\sigma\left(\pi^{+} p \rightarrow K^{+} \Sigma^{+}\right)}<\frac{0.2 \pm 0.2 \mu b}{80 \mu b} \lesssim \frac{1}{350},
$$

while at $3.55 \mathrm{GeV} / c$ (ref. [12])

$$
\frac{\sigma\left(\mathrm{K}^{-} \mathrm{p} \rightarrow \mathrm{pK}^{-}\right)}{\sigma\left(\mathrm{K}^{+} \mathrm{p} \rightarrow \mathrm{pK}^{+}\right)}<\frac{1.4 \mu \mathrm{b}}{30 \pm 1 \mu \mathrm{b}} \approx \frac{1}{20} \text {. }
$$

The upper limits quoted correspond to the cross section that would have applied if one event had been seen. In all these cases the double-exchange process could involve exchange of a charged strangeness-zero boson in addition to the type of Reggeon exchanged in the allowed reaction in the denominator.

Detection of the small double-exchange amplitude by measurement of charge ratio proves to be even more difficult. It is interesting to note that the mass of bubble chamber data on charge ratios is consistent with large deviations (say 50\%) from the predictions for no $I=2, Y=0$ and no $I=\frac{3}{2}$, $Y= \pm 1$ boson exchange. It is, on the other hand also consistent with accurate satisfaction of the predicted charge ratios. Ratios involving broad resonances are suspect and probably should not be considered unless great care has been taken in interpreting the Dalitz plot. One arm spectrometer measurements involving broad resonances may not be of value. Promising ratios to measure are

$$
\begin{aligned}
\pi^{+} \mathrm{d} & \rightarrow \mathrm{K}^{+} \Sigma^{+} \mathrm{n}, \\
& \rightarrow \mathrm{K}^{0} \Sigma^{+} \mathrm{p}, \\
& \rightarrow \mathrm{K}^{+} \Sigma^{0} \mathrm{p}, \\
\gamma \mathrm{d} & \rightarrow \mathrm{K}^{+} \Sigma^{0} \mathrm{n}, \\
& \rightarrow \mathrm{K}^{+} \Sigma^{-} \mathrm{p}, \\
\pi^{+} \mathrm{d} & \rightarrow \mathrm{K}^{+} \Sigma^{+}, \\
\pi^{-} \mathrm{p} & \rightarrow \mathrm{K}^{\mathrm{O}} \Sigma^{\mathrm{o}} .
\end{aligned}
$$

It is difficult to think of other cases; some $\mathrm{p} \overline{\mathrm{p}}$ reactions may be promising.

The ratio (10) has been measured at SLAC [18] without observing the final baryons directly. The result is a $30 \%$ deviation in rate from the $I=\frac{1}{2}$ exchange charge ratio. The result needs to be confirmed, because the method for separating the reactions is indirect.

In summary, experiments have not really settled the question of the relative size of double exchange. With the exception of two reactions (6), (7), the double-exchange amplitude might typically be $\frac{1}{5}$ the magnitude of single exchange. With the exception of one other reaction, (5), the number might be less than, or about, $\frac{1}{20}$ of single exchange. 
Theoretically, while it is difficult to predict the magnitude of double exchange, something can be said about angular distributions, and the energy dependence can be predicted. Let us now examine the theoretical situation.

The energy dependence at high energy for exchange of two Reggeons with trajectories $\alpha_{1}\left(t_{1}\right)$ and $\alpha_{2}\left(t_{2}\right)$ is, at large $s$,

$$
\sigma \propto\left|\left\langle s^{\left(\alpha_{1}\left(t_{1}\right)+\alpha_{2}\left(t_{2}\right)-2\right)}\right\rangle\right|^{2},
$$

where the average is over kinematically allowed $t_{i}$ values. It is appropriate to take the $t_{i}$ near zero, so we have the cross section decreasing as

$$
\frac{\sigma_{\text {double }}}{\sigma_{\text {allowed }}} \propto \frac{s^{2 \bar{\alpha}_{2}-2}}{\ln ^{2} s} \sim s^{-2},
$$

where the added Reggeon is assumed to be a vector or tensor boson. The double exchange cross section should not decrease rapidly, i.e., not like $\sigma \propto s^{-10}$. Instead, we imagine that $\sigma$ is large at low energies, over a short energy interval $\sigma$ decreases very rapidly to a small value, and then it follows the law (12) (ref. [19]). There is presently no confirmation of this Regge cut prediction. There is also no evidence suggesting disagreement.

The angular dependence of double exchange should, on the whole, be broader than single exchange because smaller impact parameters are involved in the former. Consider the slope $A$, in $\mathrm{d} \sigma / \mathrm{d} t \propto \mathrm{e}^{A t}$. Assume $A \approx 8$ for the Reggeon exchange input. For single exchange including absorption the average slope of the angular distribution will be between $A$ and $\frac{1}{2} A$ or about 6. For double exchange it will be between $\frac{1}{2} A$ and $\frac{1}{3} A$ or about 3.5.

The magnitude of any double exchange is hard to predict because there are many exchanges and intermediate states to consider. There are theoretical indications of strong cancellations in certain reactions [20], which may explain the variations observed in reactions (5)-(8).

We conclude that, especially at larger momentum transfer and lower energies, double exchange may be a significant correction to single exchange: about $10 \%$ in amplitude at $5 \mathrm{GeV} / c$ and probably significantly greater at large $-t$. Because the contribution is presently not known and not predictable we are forced to neglect double exchange from the rest of our considerations. But we must keep in mind that this is an approximation which may not be too good in some situations.

\section{ANGULAR DEPENDENCE OF R-TYPE REACTIONS}

\subsection{Introduction}

It is observed that $2 \rightarrow 2$ single-Reggeon exchange amplitudes often have what appears to be a simple zero as a function of $t$ or $u$ at small negative $t$ or $u$ (e.g., $-t<1 \mathrm{GeV}^{2}$ ). In this section we wish to consider several as pects of the angular distributions, especially these zeros.

The amplitudes are complex, so that for real $t$ (similar remarks apply to $u$ ) we do not expect exact zeros. We can ask, however, whether simple 
or higher-order zeros occur near the real $t$-axis in the analytic continuation of the amplitudes.

There are a number of interesting questions that should be asked about the properties of these zeros. Do amplitudes have more than one zero in $t$ as would be characteristic of an absorbing region with a sharp spatial boundary? No extensive experimental information is available, although second dips are seen in some elastic reactions. Which amplitudes exhibit the $t$-dependence most directly? What determines the presence, order and position of zeros? We will compare Regge pole models with zeros in $t$ as sociated with "nonsense factors" to a model in which zeros occur due to pole-absorptive cut interference. In particular we wish to determine whether the zeros characterizing the angular structure are due to the "Born" exchange amplitudes, or to interference with the absorption corrections made to these. We shall see that the crucial test is the helicity dependence of the structure in these two cases.

In high energy models either with or without cuts, the $s$-channel helicity amplitudes exhibit the relevant structure rather than e.g., the $t$-channel helicity amplitude or the invariant amplitudes. Consider particles with direct channel helicities $\lambda_{a}, \lambda_{b}, \lambda_{c}, \lambda_{d}$ in the c.m. frame as in fig. 1. As a consequence of angular momentum conservation, the amplitude satisfies

$$
T_{\lambda_{\mathrm{a}} \lambda_{\mathrm{b}} \lambda_{\mathrm{c}} \lambda_{\mathrm{d}}} \propto\left(t_{\mathrm{o}}-t\right)^{\frac{1}{2} n}
$$

where

$$
\begin{gathered}
t_{\mathrm{o}}=t\left(0^{\circ}\right) \underset{s \rightarrow \infty}{\longrightarrow} 0, \\
n=\left|n_{1}-n_{2}\right|, \quad n_{1}=\lambda_{\mathrm{a}}-\lambda_{\mathrm{c}}, \quad n_{2}=\lambda_{\mathrm{b}}-\lambda_{\mathrm{d}} .
\end{gathered}
$$

Consider boson exchange. (A slight modification is needed for fermion exchange.) If the amplitude connects only eigenstates of one parity in the crossed channel, then at high $s$,

$$
T_{\lambda_{\mathrm{a}} \lambda_{\mathrm{b}} \lambda_{\mathrm{c}} \lambda_{\mathrm{d}}}= \pm T_{-\lambda_{\mathrm{a}}}, \lambda_{\mathrm{b}},-\lambda_{\mathrm{c}}, \lambda_{\mathrm{d}},
$$

i.e., up to a sign it is invariant under

$$
n_{1} \rightarrow-n_{1}
$$

Single Regge-pole exchange is associated with definite parity. It follows that Regge-pole amplitudes satisfy

$$
T_{\lambda_{\mathrm{a}}, \lambda_{\mathrm{b}}, \lambda_{\mathrm{c}}, \lambda_{\mathrm{d}}} \propto\left(t_{\mathrm{o}}-t\right)^{\frac{1}{2}(n+x)},
$$

where

$$
n+x=\left|n_{1}\right|+\left|n_{2}\right|=\text { larger of }\left(\left|n_{1}-n_{2}\right|,\left|\left(-n_{1}\right)-n_{2}\right|\right) \text {. }
$$


Note that $x$ is even. The situation with $x>0$ is called "evasion".

An essential distinction between structure in $t$ due to the Regge-pole amplitudes and structure due to $s$-channel effects (absorption) is that the former structure, in particular as it depends on helicities, factors in the $t$-channel while the latter does not. Thus the Regge pole amplitude has the form $\pm c\left(\left|n_{1}\right|, t\right) c\left(\left|n_{2}\right|, t\right)$ while if $t$-dependent structure is due to $s$-channel effects (absorption) its form is $f(n, t)$ which does not factor. We will discuss how to use this factorization to distinguish which of these approaches agrees with experiment.

In the remainder of this section we will consider the absorption effects in the impact parameter representation, for $n=x=0$ and a simple Reggepole input in order to develop some qualitative notions. Then we will consider, from the standpoint of $t$-dependence, the various interesting Regge pole amplitudes, the helicity dependence in the presence of cuts, and connections with experiment.

\subsection{Qualitative $t$-dependence in the absorption model for helicity non-flip}

Consider an $n=x=0$ amplitude, i.e., no helicity change at either vertex. Let us examine the possibilities for a simple zero at small $t$ or a series of simple zeros. Experiments show a zero at $-t \approx 0.15$ to 0.25 as discussed below.

It is useful, both for pedagogical and for calculational purposes, to consider the amplitude in the impact parameter representation. The transformation between the impact parameter representation and the momentum transfer representation are

$$
\begin{aligned}
& M(t)=2 q^{2} \int_{0}^{\infty} b \mathrm{~d} b A(b) J_{0}(b \sqrt{-t}), \\
& A(b)=\int_{0}^{\infty} \frac{\mathrm{d}(-t)}{4 q^{2}} M(t) J_{0}(b \sqrt{-t}) .
\end{aligned}
$$

At high energy, a correspondence can be made with the partial wave expansion

$$
M(t)=\sum_{l}(2 l+1) a_{l} P_{l}(\cos \theta)
$$

We multiply this equation by $\delta l=1$ and make the set of approximations

$$
\begin{gathered}
l+\frac{1}{2} \approx q b, \quad \Sigma(\delta l) \approx \int q \mathrm{~d} b, \\
P_{l}(\cos \theta) \approx J_{\mathrm{o}}(b \sqrt{-t}), \quad a_{l} \approx A(b) .
\end{gathered}
$$

Although the impact parameter representation is exact at all energies, a high-energy approximation is made when conservation of angular momentum is taken to result in conservation of $b$. Thus, a double scattering which 
takes place in the same partial wave is considered to take place at the same $b$.

The amplitude in an absorption model is given in terms of a Born term $M^{P}(t)$ and the elastic $S$-matrix $S^{\mathrm{el}}$ by the Sopkovich prescription [21-24].

$$
a_{l}=s_{l}^{\mathrm{el}} a_{l}^{P}
$$

Thus we have in the impact parameter representation

$$
A(b)=S^{\mathrm{el}}(b) A^{P}(b) \text {. }
$$

This is the elastic scattering prescription for absorption.

The transformation between the impact parameter and $t$ representations is identical to the transformation in classical optics between the position on a diffracting object and the angle of the scattered light. Just as an object with a sharp edge gives rise to a diffraction pattern with minima and secondary maxima in the intensity, an "edge" in $A(b)$ will give rise to dips and secondary maxima in $|M(t)|^{2}$.

In the rest of this section we will carefully examine the properties of (18) and generalizations of (18) in impact-parameter space and in momentum-transfer space in order to understand the zeros and their positions as determined by various absorption models, assuming $A^{P}(b)$ itself is smooth and generates no zeros in $t$.

The origin of the "edge" in the absorption model is shown by the solid lines in fig. 2 (for an amplitude with $n=x=0$ ). The elastic $S$-matrix can be written as

$$
S^{\mathrm{el}}(b)=1+i T^{\mathrm{el}}(b)
$$

The term 1 represents the unscattered wave while $T(b)$ is the product of a density of states factor and the impact parameter representation of the elastic scattering amplitude. Experimentally, the elastic scattering amplitude is approximately an exponential in $t, \propto \mathrm{e}^{a t}$, with $2 a \approx 7.5 \mathrm{GeV}^{-2}$ for $\pi \mathrm{N}$. Its transform is then a Gaussian as shown in fig. $2 \mathrm{a}$ :

$$
\left|T^{\mathrm{el}}(b)\right|=\frac{\sigma_{\mathrm{T}}}{8 \pi} \mathrm{e}^{-b^{2} / 4 a} \approx 0.67 \mathrm{e}^{-0.067 b^{2}},
$$

where $\sigma_{\mathrm{T}}=25 \mathrm{mb}$ is the total cross section. Moreover, the elastic scattering is largely imaginary, so

$$
S^{\mathrm{el}} \approx 1-\left|T^{\mathrm{el}}(b)\right|,
$$

and we can rewrite eq. (18):

$$
A(b)=A^{P}(b)-\left|T^{\mathrm{el}}(b)\right| A^{P}(b) .
$$

We define the symbol $A^{C} \equiv i T^{\mathrm{el}}(b) A^{P}(b)$, approximated by the second 

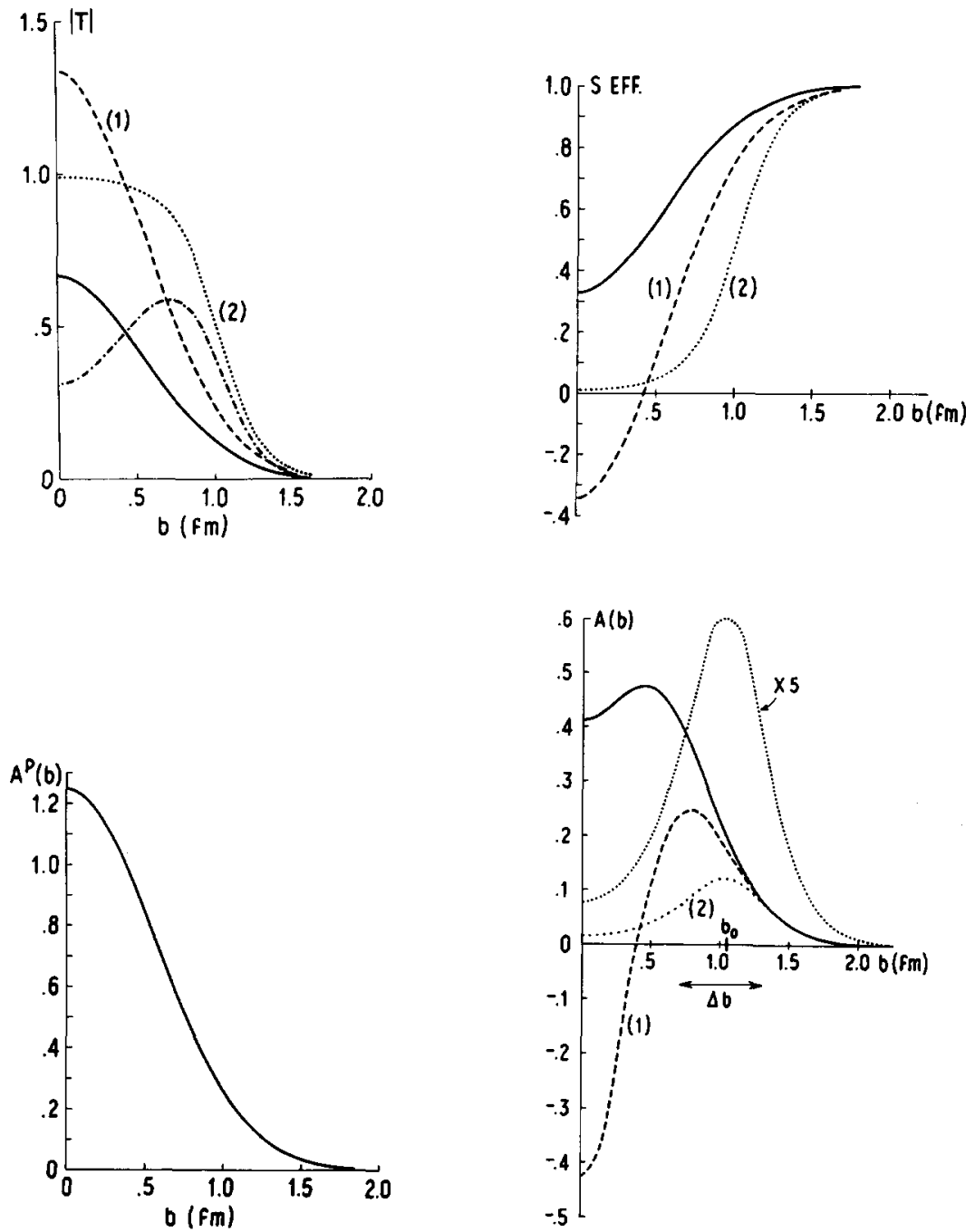

Fig. 2. The impact parameter representation of the absorption model. The solid curves are elastic absorption. The dashed curves (1) are absorption with a coherent inelastic factor $\lambda=2$. The dotted curves (2) have coherent inelastic effects included without overabsorption. (a) The effective elastic scattering amplitude. The dot-dashed curve is the inelastic contribution to (2). (b) The effective elastic $S$ matrix $S_{\text {eff }}=1-T_{\text {eff. (c) The Regge-pole input amplitude. (d) The amplitude }}$ $A(b)=A P(b) \times S$ eff. The curve $\times 5$ is curve (2) enlarged by a factor of 5 , to show its resemblence to a $\delta$-function at $b_{0}$. 
term in $(20)^{*} . S^{\mathrm{el}}(b)$ is the solid curve shown in fig. $2 \mathrm{~b}$. The Regge pole input (discussed below) is taken for the purposes of our immediate arguments roughly as an exponential in $t$ as well, so its transform is approximately Gaussian as shown in fig. 2c. In order to carry out the absorption, eq. (18), we multiply $S^{\text {el }}$ of fig. $2 b$ by the Regge pole of fig. $2 c$, and obtain the amplitude (solid curve) shown in fig. $2 \mathrm{~d}$. By comparison with the input pole amplitude, we see that the absorbed amplitude has an edge at $b \approx b_{0}$. Very roughly, the amplitude will be proportional to

$$
\int_{0}^{b} J_{\mathrm{o}}(b \sqrt{-t}) b \mathrm{~d} b=\frac{J_{1}\left(b_{\mathrm{Q}} \sqrt{-\bar{t}}\right)}{\sqrt{-t}} .
$$

This function has zeros at $b_{0} \sqrt{-t}=3.8,7.0, \ldots$ In fig. 3 we plot the amplitude from actually evaluating the transform, (still the solid curve). The first zero still appears. $b_{\mathrm{o}}$ in fig. $2 \mathrm{~d}$ has been marked so that $b_{\mathrm{o}} \sqrt{-t}=3.8$ at this zero. The higher zeros do not appear since the edge is not sufficiently sharp.

As with other transforms, an "uncertainty principle" relates the size of structure in $b$ with the size of structure in $\sqrt{-t}$. This relation is $\delta b \delta \sqrt{-t} \approx \pi$. For an edge effect there are two relevant sizes, namely the position $b_{0}$ of the edge and the thickness $\Delta b$ of the edge region. For an infinitely sharp edge, $\Delta b=0$ so that in $\sqrt{-t}$ there are structures of size $\pi / b_{0}$ and $\infty$. Thus for $\Delta b=0$ the structure of size $\pi / b_{0}$ keeps repeating ad infinitum, causing periodic zeros. For the actual curve $\Delta b>\frac{1}{2} b_{0}$. Thus there is only one zero. In general, the number of important zeros will be $b_{0} / \Delta b$.

If reasonable parameters are used, the position of the zero is found to be

$$
t \approx-0.55(\mathrm{GeV} / c)^{2} \approx-\left(\frac{3.8}{b_{0}}\right)^{2},
$$

corresponding to $b_{\mathrm{o}} \approx 1.0 \mathrm{fm}$. Experimentally the zero is at $t \approx-0.2(\mathrm{GeV} / \mathrm{c})^{2}$ corresponding to $b_{\mathrm{o}} \approx 1.7 \mathrm{fm}$. If the zero is due to absorption the explanation must be that the elastic scattering gives too small an absorption. (The necessity of increasing the absorption is implicit in the work of Gottfried and Jackson [23].)

It is reasonable to consider that the effect of absorption is significantly stronger than that given by (18). We attribute the increased absorption to the effect of diffraction inelastic scattering. Up to now, the absorption model is given by eq. (20). The first term is single scattering (fig. 4a) while the second is double scattering (fig. $4 \mathrm{~b}$ ). However, other double scatterings are possible (fig. $4 \mathrm{c}$ ). If $\mathrm{a}^{\prime}$ and $\mathrm{b}^{\prime}$ are any states with the same internal quantum numbers as $a$ and $b$, respectively, then the reaction $a+b \rightarrow a^{\prime}+b^{\prime}$ can occur by diffraction dissociation (subject to approximate selection rules), and $a^{\prime}+b^{\prime} \rightarrow c+d$ can occur by Reggeon exchange. Thus the effective absorbing

* This expression is also obtained from an eikonal approximation [25a], from a statistical approximation [25b] and from a Feynman diagram approach [25c]. The absorption approach has also been studied by L.Durand III and Y.T.Chiu (Yale University thesis, unpublished). 


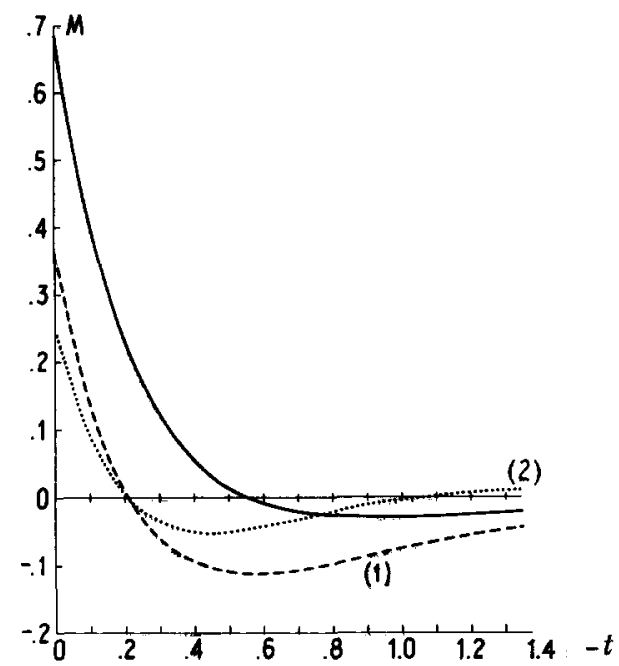

Fig. 3. The absorption model amplitude as a function of $t$. The key for the curves is the same as in fig. 2. These curves are the Hankel transform of the corresponding curves in fig. 2d. Curve (1) has one zero while curve (2) has 2 zeros. The solid curve, without coherent inelastic effects, has a zero at much larger $|t|$.

amplitude $T^{\text {eff }}$ is the sum of elastic and diffractive inelastic amplitudes, i.e., we replace (18) and (20) by

$$
\begin{aligned}
A(b) & =S^{\mathrm{eff}}(b) A^{P}(b) \\
& =A^{P}(b)-\left|T^{\mathrm{eff}}(b)\right| A^{P}(b),
\end{aligned}
$$

where

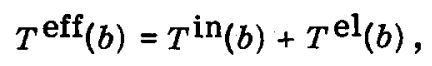

and $T^{\text {in }}$ itself is an effective amplitude defined for the two-body reaction $i \rightarrow j$ by sum over diffractive inelastic intermediate states $k$ :

$$
T^{\text {in }} A_{i j}^{P}=\sum_{k} T_{i k} A_{k j}^{P}
$$

We must include excitation of either or both particles to simple continuum states as well as resonances. Experiments indicate that Reggeon exchange to a variety of strong resonances, or to a few hundred MeV of low-mass continuum, is comparable with any particular process of this kind [26] * One finds then that the inelastic contribution to the cut amplitude $M \mathrm{C}_{(t)}$

* In ref. [26] it is shown that a single contribution to $T^{\text {in }}$ is typically 0.1 of $T^{\mathrm{el}}$. 

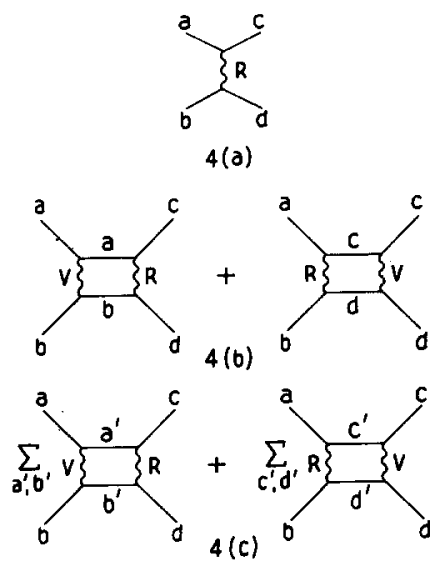

Fig. 4. Pictorial representation of absorption model terms. These are not Feynman diagrams. (a) Single Reggeon exchange. (b) Elastic absorption. V is elastic scattering. (c) Coherent inelastic absorption. $a^{\prime}$ has the same quantum numbers as a, etc.

(defined in connection with eq. (20)) is expected to be comparable to the elastic if enough contributions are included to give the total diffractive inelastic cross section, and if the sign of various inelastic contributions is the same ${ }^{*}$. One can show indeed that the contributions are relatively real and likely to be the same sign as the elastic contribution in a composite model [28]. However, the latter point has not been definitively formulated.

In previous work, we assumed that the diffractive inelastic amplitude has the same $t$ dependence as the elastic amplitude, i.e., $\left|T^{\text {eff }}\right|=\lambda\left|T^{\text {el }}\right|$. In some data fitting we adjusted the magnitude of the inelastic amplitude to be similar to that of the elastic scattering. Thus we used approximately $\left|T^{\mathrm{eff}}\right| \approx\left|2 T^{\mathrm{el}}\right|$, as shown by the dashed line labelled (1) in fig. 2a. When we carry through the absorption analysis, we get the dashed curves in figs. $2 b, 2 d$ and fig. 3 . There is a zero near $t=-0.2$ as desired. One immediately notices (figs. $2 b$ and $2 d$ ) that at small impact parameters, there is over-absorption, and the pole amplitude is multiplied by a negative number.

It is conceivable that over-absorption is physical, although contrary to one's intuition. It seems more reasonable to take another approach, by assuming that simple diffractive inelastic reactions undergo absorption, as do $R$-type inelastic reactions. Thus we should take the diffractive inelastic scattering to be reduced at small impact parameters, as shown by the dashdot curve $\left|T^{\text {in }}\right|$ in fig. 2a. The incoming flux at small impact parameter has been lost to complex, many-particle inelastic processes. It is highly im-

\footnotetext{
* This is a crude estimate. There is no indication that this can be directly determined in the near future from direct examination of simple diffractive inelastic processes (as opposed to determining $M^{\mathrm{C}}$ through application of the absorption model). Note that the sum of all simple diffractive inelastic cross sections is, within a factor of two, half the elastic cross section [27].
} 
probable that the resultant particles from a highly inelastic process can be recombined to form a final two-particle state. Thus, the inelastic contribution to $T^{\text {eff }}$ should be concentrated at the "surface" because the simple inelastic states should make the dominant contributions. Adding $T^{\text {in }}$ to elastic scattering we get the effective absorbing amplitude $T^{\text {eff }}$ of fig. 2 a (dotted curve, labelled 2). Carrying out the absorption we get the dotted curves labelled 2 in fig. $2 b$ and $2 d$, and fig. 3.

The particular curves (2) were calculated for illustrative purposes as follows. Assume the simple inelastic processes that contribute are governed by the absorption model under discussion, i.e., for simple inelastic processes, at a given $b$

$$
\left|T^{\text {in }}\right|=|B|-\left|T^{\text {eff }}\right||B|
$$

or

$$
\left|T^{\mathrm{in}}\right|=\frac{|B|}{1+|B|}\left(1-\left|T^{\mathrm{el}}\right|\right) .
$$

For simplicity we take the driving term $|B|=C\left|T^{\mathrm{el}}\right|^{2}$. In the figures (dotted curves labelled 2) $C=56$, and $T^{\mathrm{el}}$ is the Gaussian discussed above eq. (20), so that

$$
\left|T^{\text {in }}\right|_{\text {small } b}^{\approx}\left(1-\left|T^{\mathrm{el}}\right|\right)=S^{\mathrm{el}},
$$

and where for $b$ sufficiently large so that $|B| \ll 1$

$$
\left.\left|T^{\text {in }} \underset{\text { large } b}{\approx}\right| B|=C| T^{\mathrm{el}}\right|^{2} \text {. }
$$

The curve for $\left|T^{\text {in }}\right|$ is the dot and dashed curve of fig. 2a. We note that $T^{\text {in }}$ and $T$ el are comparable in magnitude in conformity with the discussion above.

In the two cases with strong absorption the total amount of effective absorption is about the same. (See fig. $2 a$ or $2 b$.) Since the curve (2) is smaller than the curve (1) at small impact parameters in fig. 2a, it must therefore extend farther out. This gives rise to a sharper edge, that is, a smaller $\Delta b$. Therefore, more zeros are generated in the amplitude (2) as a function of $t$. For the particular curves (2) and (1) of fig. $2 \mathrm{~d}$ we get either two or one zero, as shown in fig. 3. As can be seen, the difference between the two cases is at larger $|t|$, where the cross section is smaller, and therefore not so well determined experimentally. Thus our previous successful phenomenology $[1,29,30]$ with an overabsorbing effective amplitude does not imply that overabsorption is physical.

If only single scattering were important, there would be no zeros of the type we are discussing. By considering double scattering, we get one zero. Including absorption for diffractive scattering, and thus taking triple scattering (i.e., through eq. (23)) into account we get a sharper edge, which can lead to two zeros. For cases in which higher-order scattering becomes important, we expect even more zeros. Thus in nuclear physics many orders of scattering are needed so that the total absorption at small impact param- 
eters is almost exactly $100 \%$. As a result, the edge can become quite sharp, and many diffraction minima can be seen as a function of momentum transfer.

We conclude: a diffraction like pattern in $t$ can be an edge effect. The edge is not apparent in the actual matter or opacity distributions, but rather is generated through inelastic reactions in a multiple scattering process. The inelastic intermediate states in double scattering play two roles: They strengthen the cut, or absorption corrections, increasing the radius characterizing the diffraction pattern, and they can sharpen the surface definition leading to a diffraction pattern which may have more than one zero.

\subsection{The Regge pole amplitude}

As stated above the observed zeros in inelastic angular distributions require either that the Born term (the Regge pole exchange amplitude $M^{P}$ ) has these zeros at roughly the observed positions, or that the absorption correction factor $S \operatorname{eff}(b)$ has a fairly well defined edge. We now review the way in which zeros might enter directly through the Born term. There is consider able freedom; however, the constraint of factorization should be powerful enough to resolve the question by comparison with data.

In conventional Regge pole models the full amplitude has a factor

$$
\frac{1}{\sin \pi \alpha(t)} \text {. }
$$

There are a variety of numerator factors to eliminate the unwanted poles of $t$ in (24): signature zeros, e.g., $\sin \frac{1}{2} \pi \alpha$ if the particle spins are odd, nonsense zeros, other ghost eliminating zeros, etc. There is no agreed procedure to fix all these factors [31-33] or to determine further dependences such as additive or multiplicative fixed poles. In the conventional formulations when two of these numerator factors vanish at the same value of $t$, with $\alpha(t)=$ integer, the amplitude does not merely lack a pole, it vanishes! Of the variety of conventional cases two are, perhaps, interesting enough to exhibit.

We will consider three Regge pole models, including one unconventional one which will be discussed below. The other two are a model, $\mathrm{N}$, with zeros in flip amplitudes as required by "nonsense" arguments but not in non-flip amplitudes where not so required, and a model $\mathrm{V}$ with zeros in both flip and non-flip amplitudes where a zero is required in the flip amplitude. Model $\mathrm{N}$ has been the most popular model among Regge polologists. Model $\mathrm{V}$ has been suggested by exchange degeneracy arguments $[34,35]$. The Veneziano model [36] is of this type.

Let us work in terms of the following example: the magnitudes of the helicity changes at each vertex, $\left(n_{1}, n_{2}\right)$ are $(0,0),(0,1)$ and $(1,1)$. Consider $\alpha\left(t_{1}\right)=0$. The zeros at $t=t_{1}$ are given in table 2 with the two conventional Regge models of interest in columns two and three, Near $t_{1}$, the net amplitude is a complex constant times the factor shown, It is seen that factorization applies. In the model $N$ there is a factor $\left.\alpha\left|n_{1}\right|+\left|n_{2}\right|\right)$. In the model $\mathrm{V}$, every amplitude vanishes linearly at $t=t_{1}$.

Several authors [37-39] have shown that these zeros are not necessary. 
Table 2

Behaviour at $\alpha=0$ in various Regge-pole amplitudes.

\begin{tabular}{ccccccc}
\hline & Helicities & \multicolumn{3}{c}{ Regge-pole model } \\
\cline { 2 - 6 }$n_{1}$ & $n_{2}$ & $n$ & $\mathrm{~s}$ & $\mathrm{~N}$ & $\mathrm{~V}$ \\
\hline 0 & 0 & 0 & 1 & 1 & $\alpha$ \\
1 & 0 & 1 & 1 & $\alpha$ & $\alpha$ \\
1 & -1 & 0 & 1 & $\alpha^{2}$ & $\alpha$ \\
\hline
\end{tabular}

If there are cuts in the $j$-plane the amplitude will generally have fixed poles at nonsense, wrong signature values of $j$. It is to be expected that these poles interact with Regge poles in a partly multiplicative way. If the partial wave amplitude has a fixed multiplicative pole at, e.g., $j=0$, $a_{j} \approx(j[j-\alpha(t)])^{-1}$, then the zero is removed. (The interesting theoretical exercise of studying scattering of bound two-body systems on each other in order to determine $j$-plane behaviour in Schroedinger theory, has not, to our knowledge, been done.) There is, thus, another Regge pole model which we call "choosing simplicity", [32] which is most conveniently expressed by explicitly representing the poles in $t$ instead of (24), e.g., by

$$
P=\sum \frac{C_{r}}{r-m_{r}^{2}}
$$

With this form, no factors are required to compensate for unwanted poles. This model has no zeros at $t_{1}$ and is represented under the column $\mathrm{S}$ in table 2.

To summarize, we have the full $s$-channel helicity amplitudes for Reggeon exchange [40] (leading term at large $s$ ).

Conventional [31]:

$$
M^{P}=(-t)^{\frac{1}{2}(n+x)} c_{n_{1}}(t) c_{n_{2}}(t) \frac{g_{n_{1}}(t) g_{n_{2}}(t)}{\sin \pi \alpha} \mathrm{e}^{-\frac{1}{2} i \pi\left(j_{1}-\alpha\right)} \epsilon\left(\frac{s}{s_{\mathrm{o}}}\right)^{\alpha(t)} .
$$

Simplicity :

$$
M^{P}=(-t)^{\frac{1}{2}(n+x)} g_{n_{1}}(t) g_{n_{2}}(t) P(t) \mathrm{e}^{-\frac{1}{2} i \pi\left(j_{1}-\alpha\right)}\left(\frac{s}{s_{0}}\right)^{\alpha(t)},
$$

where the $g_{n}$ are factorized residue functions which do not have zeros, $\epsilon$ is a signature coefficient, e.g., $\sin \frac{1}{2} \pi \alpha$ for odd spins, and $j_{1}$ is the spin of the lowest mass particle on the trajectory. With $c_{n_{1}}(t) c_{n_{2}}(t)$ we explicitly represent the $t$-dependent factors shown in table 2. Similar factors may arise for $\alpha=-1,-2 \ldots$ In (27) $P$ is the propagator factor (25) having a pole at every particle on the trajectory with residues of alternating sign. For example $P=\Gamma\left(\frac{1}{2}\left(j_{1}-\alpha(t)\right)\right.$ or $P \approx\left(t-m^{2}\right)^{-1}$ where $m$ is the mass of the lowest-mass particle on the trajectory. Thus in (27) $M^{P}(t)$ has no zeros for $-t>0$ if the $g$ have no zeros. The equivalent amplitude for fermions can be found in Kelly et al. [29]. 


\subsection{Form of the helicity amplitudes}

We now add absorption effects to the Regge pole input discussed above. For $n_{1}=n_{2}=0=n=x$ we have

$$
M(t)=\sum_{l}(2 l+1) a_{l}^{P} S^{\mathrm{eff}} P_{l}(\cos \theta)
$$

For the general case we have

$$
M(t)=\sum_{j}(2 j+1) a_{j}^{P} s_{j}^{\text {eff }} d_{h_{1} h_{2}}^{j}(z) .
$$

Note that $S$ is assumed to be helicity conserving at each vertex so that the helicity labels on $M$ are the same as those on $M^{P}$. Here $h_{1}=\lambda_{\mathrm{a}}-\lambda_{\mathrm{b}}$, $h_{2}=\lambda_{\mathrm{c}}-\lambda_{\mathrm{d}}$. The impact parameter version of $(28)$ is

$$
M=2 q^{2} \int b \mathrm{~d} b A^{P}(b) S^{e f f}(b) J_{n}(b \sqrt{-t}) .
$$

If the simple pole (27) with no zeros for $-t>0$ is adopted and the numerical integration (28) carried out, with reasonable parameters, we obtain the sketches shown in figs. $5 \mathrm{a}, \mathrm{b}, \mathrm{c}, \mathrm{d}$. We consider in these four drawings the

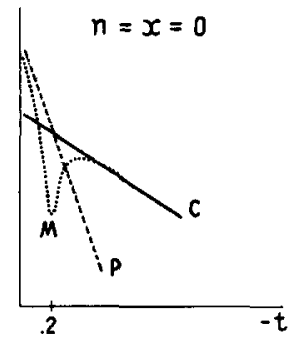

(a)

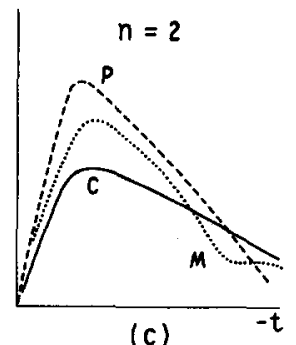

(c)

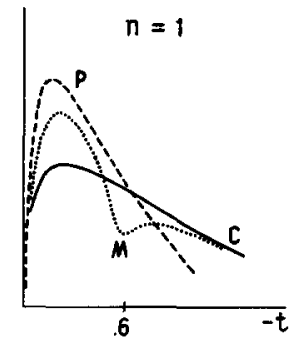

(b)

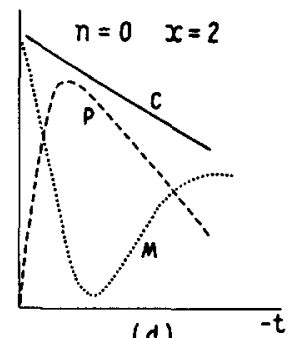

(d)

Fig. 5. Magnitudes of input Regge-pole amplitude ( $P$, dashed curve), absorption correction (Regge-cut $C$, solid curve), and their sum (M, dotted curve) for various values of $(n, x):(a),(0,0) ;(b),(1,0) ;(c),(2,0) ;(d),(0,2)$. The interference is primarily destructive leading to dips. The approximate positions of the dips (in $\left.(\mathrm{GeV} / c)^{2}\right)$ is shown for the two most important cases. 
Table 3

Position of zeros in helicity amplitudes in the approximation

$$
M_{n}(t) \propto J_{n}\left(b_{0} \sqrt{-t)} \text { with } b_{0}=1 \mathrm{fm}\right. \text {. }
$$

\begin{tabular}{llccc}
\hline Zero & $n$ & 0 & 1 & 2 \\
\hline First & 0.23 & 0.59 & 1.06 \\
Second & & 1.22 & 1.97 & 2.83 \\
Third & 3.00 & 4.14 & 5.40 \\
\hline
\end{tabular}

Values of $-t$ in $\mathrm{GeV}^{2}$ are shown.

same helicity amplitudes as above in table $2:\left(n_{1}, n_{2}\right)=(0,0),(0,1)$ and $(1,1)$, $n=0$ and 2. The results for $x=0$ (e.g., excluding the $(1,1) n=0$ example) can be summarized analytically by approximating (28): If $b A^{P}(b) S^{\operatorname{eff}_{(b)} \text { is }}$ sharply peaked at $b_{0}$ as approximated by curve 2 , fig. $2 \mathrm{~d}$, we can approximately replace it with a delta function and obtain

$$
M(t) \approx \text { const } \times J_{n}\left(b_{0} \sqrt{-t}\right) \quad \text { for } \sqrt{-t}<\frac{\pi}{\Delta b},
$$

where, again, $n=\left|h_{1}-h_{2}\right|=\left|n_{1}-n_{2}\right|$ and $\Delta b$ is the width of the peak. If $b_{0}=1 \mathrm{fm}$, the zeros of each helicity amplitude would occur in this approximation at the values of $t$ shown in table 3. It is seen that the first zero for $n=0$ and 1 agree well with experiment. The others are not yet observed. Unfortunately (the theory predicts that) zeros at larger $t$ are less well defined (farther from the real $t$ axis) because $\Delta b \neq 0$ and the relative phase of pole and cut are growing away from $\pi$ as $-t$ gets larger, so they may be hard to observe. We note that the difference between (21) and (29) for $n, x=0,0$ is due to the difference between a uniform amplitude for $b<b_{0}$ and an amplitude concentrated at $b_{0}$. The values of $b_{0}$, appropriate to the two cases as shown in figs. 2 and 3 , are essentially the same, $b_{0} \approx 1.05 \mathrm{fm}$.

The important result of absorptive cuts with "simple" Regge pole input is that every s-channel helicity amplitude, with $x=0$, has a zero (or series of zeros) which move to larger-t with the parameter $n$, the net helicity flip. If the cut, or the absorption, is made roughly twice as strong as indicated by elastic absorption the nearest zeros are roughly at $-t \approx 0.2,0.6,1.2$ for $n=0,1,2$ respectively in accordance with $b_{0} \approx 1 \mathrm{fm}$ in (30). We title the model with simple Regge pole and strong absorption cut, adjusted to obtain zeros at roughly these $t$, the strong-cut Reggeized absorption model, or SCRAM. Thus the parameters of the absorption prescription are to be constrained to yield zero structure roughly as given by (30).

We expect variation in the position of zeros with the range of forces and the range of absorption. Consider eq. (30) and $b_{0} \approx \frac{1}{2}\left(r_{\mathrm{R}}+r_{\mathrm{V}}\right)$ where $r_{\mathrm{R}}$ and $r_{V}$ are radii for Reggeon exchange and diffraction, respectively. For mesonbaryon scattering with vector meson exchange $r_{V} \approx r_{R} \approx 1 \mathrm{fm}$. In baryonbaryon scattering, $a \approx 10 \mathrm{GeV}^{-2}$, about $\frac{1}{3}$ larger than for $\pi \mathrm{N}$ scattering. Since $a \propto r \overline{\mathrm{V}}^{2}$, this implies roughly a $15 \%$ decrease in $-t_{1}$, the position of a zero for any $n$, Variations in the Regge-pole amplitude may be expected for 
Table 4

Position and order of zeros in pole and pole plus cut amplitudes for $-t \lesssim 1 \mathrm{GeV}^{2}$ for the three inputs of table 2 .

\begin{tabular}{ccccccccc}
\hline & \multicolumn{3}{c}{ Helicities } & \multicolumn{5}{c}{ Position and order of zeros in $-t$ in $\mathrm{GeV}^{2}$} \\
$\left|n_{1}\right|$ & $\left|n_{2}\right|$ & $n$ & $x$ & SCRAM & $\begin{array}{c}\text { pole only } \\
\text { N }\end{array}$ & $\mathrm{V}$ & $\mathrm{N}$ & $\mathrm{V}$ \\
\hline 0 & 0 & 0 & 0 & 0.2 & none & 0.6 & 0.55 & 0.35 \\
1 & 0 & 1 & 0 & 0.6 & 0.6 & 0.6 & 0.5 & 0.5 \\
1 & 1 & 2 & 0 & 1.2 & $(0.6)^{2}$ & 0.6 & $(0.6)^{2}$ & 0.6 \\
1 & 1 & 0 & 2 & $(0.1)^{2}$ & $0,(0.6)^{2}$ & $0,0.6$ & $0.02,0.4,0.9$ & $0.03,0.5$ \\
\hline
\end{tabular}

A strong-cut is added to the simple pole (SCRAM). Elastic absorptive cuts are added to $\mathrm{N}$ - and $\mathrm{V}$-poles. We consider a meson-baryon reaction with vector meson exchange with $\alpha(-0.6)=0$. The notation $(0.6)^{2}$ refers to two nearby zeros or a double zero. Other parameters used in the numerical work are $M^{P}=\exp (4 t)$ and $T^{\mathrm{el}}=0.67 \exp$ $(3.75 t)$.

* Zeros may be distant from real axis.

different kinds of $s$-channel particles and exchanged trajectories. For any but the exchange of a $\pi$ we might expect perhaps $50 \%$ variations in $r_{\mathrm{R}}^{2}$ corresponding to $25 \%$ variations in $t_{1}$. In other words we might locate the $(n, x)=(0,0)$ zero at $0.2 \pm 0.05(\mathrm{GeV})^{2}$ and the $(n, x)=(1,0)$ zero at $0.6 \pm 0.15(\mathrm{GeV} / c)^{2}$.

The conventional Regge-pole model, $\mathrm{N}$, has zeros that factor according to $n_{1}$ and $n_{2}$, the helicity flip at each vertex, so their presence and position is not dictated by $n$. Adding absorptive cuts to these pole amplitudes will move the first zero in the pole amplitude to smaller $-t$ or introduce a zero, as in the simplicity case, where there is no zero in the pole amplitude.

In table 4 we summarize numerical results for positions of zeros for the various models discussed above. In the conventional models one can claim to have the choice between the zero structure exhibited as "pole only" or, as in the final columns, with elastic cuts, or with strong-cuts. From table 4 the patterns of zeros in these cases are clear *.

Zeros which arise from absorption effects depend on the $s$-channel helicity indices $(n, x)$. This result, e.g., dependence on $n$ in eq. (30), is independent of the detailed way in which one calculates an absorption correction. On the other hand zeros which arise from the underlying exchange mechanism factor in the $t$-channel. These zeros depend on helicity changes $n_{1}$ and $n_{2}$ at the two vertices separately. Broadly speaking our task is now to de-

* We have taken vector meson exchange as an example. If a similar table were prepared for another exchange, the results would be the same for SCRAM. The only exception is pion exchange, with its pole very close to the scattering region, which would be different. In particular for $n=0 \pi$ exchange the first zero in SCRAM is roughly at $-t=0.1$ and the first zero for $n=1$ is near $-t=0.4$. For conventional Regge poles, the entries depend critically on the nature of the exchanged Reggeon. 
termine whether this factorization is observed experimentally, or whether instead the dependence on $n$ is correct.

\subsection{Comparison with experiment}

In table 5 we show the various combinations of helicities which can occur in the reactions (forward)

$$
\begin{aligned}
\mathbf{P S}+\mathrm{B} & \rightarrow \mathbf{P S}+\mathbf{B}, \\
& \rightarrow \mathbf{P S}+\mathbf{B}^{*}, \\
& \rightarrow \mathbf{M}+\mathbf{B}, \\
& \rightarrow \mathbf{M}+\mathbf{B}^{*}, \\
\mathbf{B}+\mathrm{B} & \rightarrow \mathrm{B}+\mathbf{B}, \\
& \rightarrow \mathrm{B}+\mathbf{B}^{*},
\end{aligned}
$$

and backward

$$
\begin{aligned}
\mathrm{PS}+\mathrm{B} & \rightarrow \mathrm{B}+\mathrm{PS}, \\
& \rightarrow \mathrm{B}+\mathrm{M},
\end{aligned}
$$

where

$$
\begin{aligned}
\text { PS } & =\text { pseudoscalar } \\
\mathbf{M} & =\text { vector or tensor meson, } \\
\mathbf{B} & =\text { spin } \frac{1}{2} \text { baryon, } \\
\mathrm{B}^{*} & =\text { spin } \frac{3}{2} \text { baryon. }
\end{aligned}
$$

Reactions $\mathrm{B}+\mathrm{B} \rightarrow \mathrm{B}^{*}+\mathrm{B}^{*}$ should also be considered.

The qualitative structure of the helicity amplitudes at small momentum transfer, particularly with respect to zeros, is evident from the examples given in the preceding section, table 4 , and fig. 5 for three different pole and cut models. Note that the parity argument made to define $x$ for forward reactions is quite complicated for baryon exchange. After the arguments are made the effective value of $x$ is zero for $\left|n_{1}\right|=\left|n_{2}\right|=\frac{1}{2}$, as shown in table 5 with asterisks ${ }^{\dagger}$.

One type of comparison of theory with experiment is a full quantitative fit of a given model to many experiments. We are making such a comparison of SCRAM with experiment in other articles $[1,29,30]$. This effort involves fine adjustment of many elements of the model: coupling strengths, pole shapes, the form for $S_{\text {eff }}$ in calculating the cuts. Although most param. eters in such a theory are approximately known, the task of making an accurate comparison is formidable because the number of parameters is very large. The parameters occur in groups requiring simultaneous comparison with $\approx 6$ reactions in order that the number of parameters per reaction be 
Table 5

(a) Examples of different values of $(n, x)$ for the same $\left(n_{1}, n_{2}\right)$.

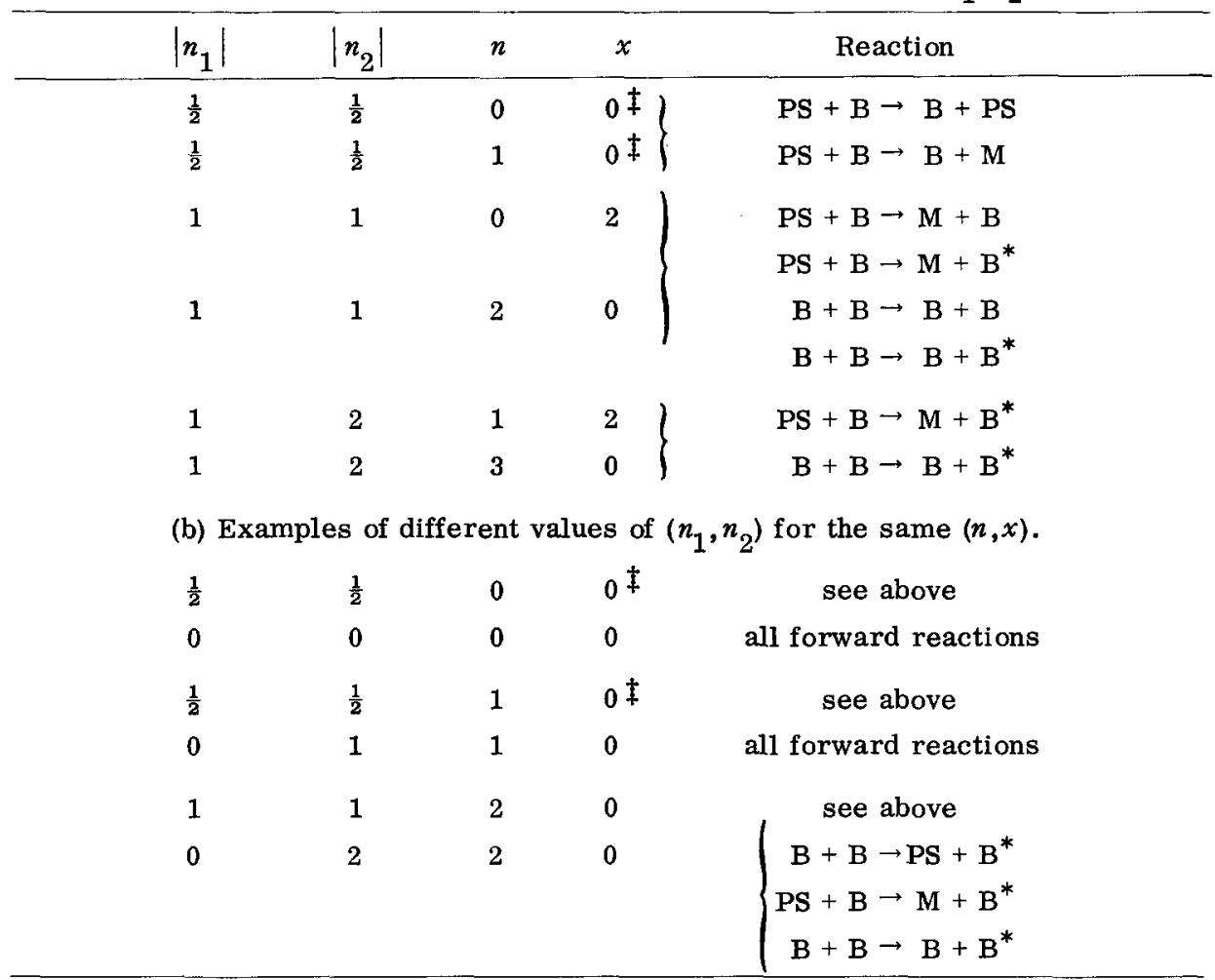

$¥$ See text for determination of $x$ for backward reactions.

† The simple partity argument and the resulting definition of $x$ that we have given applies only in the case in which the Toller number $M$ equals zero. In SCRAM, higher $M$-values for forward reactions are rejected as unnecessary complications, and therefore the existence of conspiring trajectories for $M \geqslant 1$ is regarded as a highly unlikely coincidence.

For backward meson-baryon reactions, the Toller number is a half-integer as a consequence of MacDowell symmetry. Thus the smallest $M$ and simplest case is $M=\frac{1}{2}$. For general $M$,

where

$$
x+n=\left|n_{1}^{\prime}\right|+\left|n_{2}^{\prime}\right|
$$

$$
\begin{aligned}
& n_{1}^{\prime}=\left|n_{1}\right|-M, \\
& n_{2}^{\prime}=\left|n_{2}\right|-M .
\end{aligned}
$$

If this formula gives $x<0$, a cancellation between the two amplitudes of opposite parity takes place, and the effective value of $x$, after these cancellations, is $x_{\text {eff }}=0$. In table 5 we use the effective $x$-values for backward reactions (taking $M=\frac{1}{2}$ ). 
about 4. A different type of comparison of a model with experiment which has been tried by others involves variation of only a few parameters, with many others determined by assumption (e.g., via symmetry models [41-44], or via low energy experiments [45]). Depending on the detailed work done, such comparisons can be made with individual experiments. They are more or less quantitative. In the present paper we do not propose any detailed fit to data; rather we compare theory with distinctive qualitative features that are evident in a few of the experiments.

The most interesting experiments can be conveniently grouped according to evidence they bring to bear on $(1)$ possible zeros near $-u$ or $-t \approx 0.2$, (2) possible zeros near $-t=0.6$, and (3) the structure near $t \approx 0$ for $\pi$-exchange. All these tests are for $n \leqslant 1$. Although there are distinctive features in various theories for $n>1$, it appears difficult experimentally to obtain direct information on $n>1$ amplitudes, simply because processes having $n>1$ amplitudes also have many $n \leqslant 1$ amplitudes.

Structure at $-t,-u \approx 0.2$

Theory: Of all Regge-pole inputs, only the conventional nucleon exchange has a nonsense-wrong signature zero in the region of interest $(u \approx-0.15)$. This zero occurs in all helicity amplitudes. Other zeros can occur in conventional models through accidental vanishing of the residue function. In SCRAM zeros near $-t$ or $-u=0.2$ can only arise from cut-pole interference for $(n, x)=(0,0)$. As stated above, for $(n, x)=(0,0)$ the elastic absorption model without an input zero (model $\mathrm{N}$ ) will yield a zero at roughly $t$ or $u=-0.5$. The exact position depends on pole amplitude shape and the assumed elastic amplitude. However, to obtain a zero at $t$ or $u \approx-0.15$ to -0.2 the cut must be $50 \%$ to $100 \%$ stronger at these small momentum transfers. In model $\mathrm{V}$ with an input zero at $-t=0.6$, for $(n, x)=(0,0)$, elastic absorption places the zero at about $-t=0.35$ for meson-baryon scattering and at about 0.30 for baryon-baryon scattering $[46,47]$.

Experiment: Elastic differential crossovers give the best evidence on an $(n, x)=(0,0)$ zero [48]. The difference between differential cross sections with one particle charge-conjugated is equal to the interference of a particle exchange amplitude with the vacuum exchange amplitude, $A^{\mathrm{V}}$. The latter is assumed to be essentially imaginary and helicity non-flip at small momentum transfers.

$$
\begin{aligned}
& \Delta_{\pi} \equiv \frac{\mathrm{d} \sigma}{\mathrm{d} t}\left(\pi^{-} \mathrm{p} \rightarrow \pi^{-} \mathrm{p}\right)-\frac{\mathrm{d} \sigma}{\mathrm{d} t}\left(\pi^{+} \mathrm{p} \rightarrow \pi^{+} \mathrm{p}\right)=2 \operatorname{Re} \sum_{\lambda^{\prime} \mathrm{s}} A_{\pi \mathrm{N}}^{\rho} A_{\pi \mathrm{N}}^{\mathrm{V}^{*}} \\
& \approx 2\left[\operatorname{Im} A_{\pi \mathrm{N}}^{\rho}(n, x=0,0)\right]\left|A_{\pi \mathrm{N}}^{\mathrm{V}}\right| \\
& \Delta_{\mathrm{K}} \equiv \frac{\mathrm{d} \sigma}{\mathrm{d} t}\left(\mathrm{~K}^{-} \mathrm{p} \rightarrow \mathrm{K}^{-} \mathrm{p}\right)-\frac{\mathrm{d} \sigma}{\mathrm{d} t}\left(\mathrm{~K}^{+} \mathrm{p} \rightarrow \mathrm{K}^{+} \mathrm{p}\right)=2 \operatorname{Re} \sum_{\lambda ' \mathrm{~s}}\left[A_{\mathrm{KN}}^{\rho}+A_{\mathrm{KN}}^{\omega}\right] A_{\mathrm{KN}}^{\mathrm{V}^{*}} \\
& \approx 2 \operatorname{Im}\left[A_{\mathrm{KN}}^{\rho}+A_{\mathrm{KN}}^{\omega}\right]_{n, x=0,0}\left|A_{\mathrm{KN}}^{\mathrm{V}}\right| \text {, }
\end{aligned}
$$




$$
\begin{aligned}
\Delta_{\mathrm{N}} \equiv \frac{\mathrm{d} \sigma}{\mathrm{d} t}(\overline{\mathrm{p}} \mathrm{p} \rightarrow \overline{\mathrm{p}} \mathrm{p})-\frac{\mathrm{d} \sigma}{\mathrm{d} t}(\mathrm{pp} \rightarrow \mathrm{pp}) & =2 \operatorname{Re} \sum_{\lambda ' \mathrm{~s}} A_{\mathrm{NN}}^{\omega} A_{\mathrm{NN}}^{\mathrm{P}^{*}} \\
& \approx 2\left[\operatorname{Im} A_{\mathrm{NN}}^{\omega}(n, x=0,0)\right]\left|A_{\mathrm{NN}}^{\mathrm{P}}\right| .
\end{aligned}
$$

According to all the models we are considering, the phase of the Reggeon exchange amplitude for small $-t$ is $\frac{1}{2} \pi(1-\alpha) \bmod \pi$ with $\alpha$ for the $\rho$ and $\omega$ roughly 0.3 near $t=-0.2$, so the phase is not $\frac{1}{2} \pi$. In all these models, the vanishing of the cross section differences (33)-(35) requires that the magnitude of $A_{\pi \mathrm{N}}^{\rho},\left[A_{\mathrm{KN}}^{\rho}+A_{\mathrm{KN}}^{\omega}\right]$, and $A_{\mathrm{NN}}^{\omega}$ vanish. The best evidence on the values of $t$ at which these cross sections are equal is, in our judgement,

$$
\begin{aligned}
\Delta_{\pi}=0 & \text { at }-t=0.2, \\
\Delta_{\mathrm{K}}=0 & \text { at }-t=0.1 \text { to } 0.2, \\
\Delta_{\mathrm{N}}=0 & \text { at }-t=0.2 .
\end{aligned}
$$

If these zeros are not to be explained in terms of strong-cuts, the residues must accidentally vanish. (In case $\mathrm{V}$, there would then be an extra zero at 0.6 for which there is no evidence.) This assumption leads, via factorization, to unsatisfactory predictions that other amplitudes with $n_{1}$ or $n_{2}=0$ vanish [49]. Thus we conclude these zeros must be due to interference of the pole with a strong-cut.

The other definitive experimental evidence for a zero near $-t,-u \approx 0.2$ is the dip in $\pi^{+} \mathrm{p} \rightarrow \mathrm{p} \pi^{+}$at $u \approx-0.15$ (refs. [50, 51]) shape of the differential cross section for $\theta \rightarrow 180^{\circ}$ shows that the $n=1$ helicity amplitude, which contains a factor $\left.\sqrt{(} u_{\mathrm{O}}-u\right)$ or $\sin \theta$ is very small compared to the $n=0$ amplitude. Thus either in $\operatorname{SCRAM}(n, x)=(0,0)$ dominates and there is a diffraction zero near $u \approx-0.2$, or in the conventional Regge models $\alpha_{N}=-\frac{1}{2}$ so that there is a nonsense wrong-signature zero near this point. Both agree with experiment.

A very similar situation exists for $\mathrm{K}^{-} \mathrm{n} \rightarrow \Lambda \pi^{-}$(ref. [52]). A deep dip is observed near $-u \approx 0.25$ and the shape of the differential cross section for $\theta \rightarrow 180^{\circ}$ shows that $n=0$ dominates. (Consistent with $n=0$ dominance in $\pi^{+} \mathrm{p} \rightarrow \mathrm{p} \pi^{+}$.) Because of the deuteron target $u\left(180^{\circ}\right)$ varies a little from event to event. One must avoid the trap of looking for the $\theta \rightarrow 180^{\circ}$ behaviour in the $u$-plot ${ }^{*}$. The $\theta$, rather than the $u$-behaviour must be examined directly. We also note that the observed polarization is consistent with an order of magnitude difference between the contribution of $n=0$ and 1 to the cross section.

* Drago et al. (RHEL preprint, revised) assume incorrectly that the $\mathrm{K}^{-} \mathrm{n} \rightarrow \Lambda \pi^{-}$data provide evidence for spin-flip dominance. On the contrary, the data actually provide evidence for spin non-flip dominance (Kwan Lai, private communication), consistent with other considerations (see subsect. 3.6 prediction 6 , below).

Drago et al. claim that SCRAM could have the flexibility to yield a zero at $-u \approx 0.2$ in the spin-flip amplitude. On the contrary, we have explicitly defined SCRAM in this paper to avoid such manipulations. The example of Drago et al. exceeds reasonable constraints on the parameters. 
The shape of $d \sigma / d \Omega$ near $180^{\circ}$ is a much more sensitive test of $n=0$ dominance.

The question about other backward reactions can then be asked: Do other nucleon and $\Delta$-exchange amplitudes vanish near this same point? The conventional Regge models predict that both $n=0$ and 1 nucleon exchange amplitudes vanish and that neither $\Delta$-exchange amplitude vanishes. SCRAM predicts that both $\mathrm{N}$ and $\Delta n=0$ amplitudes vanish, but that neither $\mathrm{N}$ nor $\Delta n=1$ amplitude vanishes near $u=-0.15$. The most definitive experimental test so far concerns the possible zero for $n=1$ with nucleon exchange, i.e., does the full nucleon exchange (cross section) vanish or does only the $n=0$ part of it vanish? If all helicity amplitudes for nucleon exchange vanish at $\alpha=-\frac{1}{2}$ then any reaction dominated by nucleon exchange must show a dip at $u \approx-0.15$. One can make a series of arguments based on factorization for the Regge poles which, if they are all accepted, show that this prediction disagrees with current data on other backward reactions (Kelly et al., [29]).

The only other reaction where a serious claim has been made that a zero has been separately observed near $-t=0.2$ is $\pi^{-} \mathrm{p} \rightarrow \eta \mathrm{n}$. Here observation of a zero at $t \approx-0.2$ is claimed for $(n, x)=(0,0)$ using finite energy sum rules [53], as expected in SCRAM, but not in conventional models.

Conclusion: There is ample evidence that $(n, x)=(0,0)$ amplitudes have a zero near 0.2 which can be reasonably explained only by cut-pole interference. Strong-cuts are needed to put this zero at the small momentum transfer where it is observed. This is consistent with evidence that the $n=1 \mathrm{nu}$ cleon exchange amplitude does not vanish at $\alpha=-\frac{1}{2}$, i.e., it does not have the nonsense wrong signature zero.

\section{Structure at $t \approx-0.6$}

Theory: Conventional models attribute zeros at $t \approx-0.6$ of vector meson exchange reactions to $\alpha(t)=0$ for the vector meson trajectory [54]. As table 2 shows, in model $\mathrm{N}$ the zero is of the type $\alpha^{\left|n_{1}\right|} \alpha^{\left|n_{2}\right|}$ (nonsense wrongsignature zero), while for model $\mathrm{V}$ there is a simple zero at $\alpha=0$ in all helicity amplitudes. On the other hand, SCRAM predicts a zero at $-t \approx 0.6$ for $(n, x)=(1,0)$ regardless of the type of exchange and not for any other $(n, x)$ values. Although tensor meson exchange provides distinctive predictions, at present we cannot suggest any reaction where these predictions can be tested, so we concentrate in the following on vector meson exchange.

We need to seek out differential cross sections dominated by either $\rho$ - or $\omega$-exchange. From the vector dominance analyses of the nucleon form factors, the $\rho$ and $\omega$ are dominantly coupled with $\left|n_{1}\right|=1,0$, respectively, at a baryon vertex. The differential cross sections of interest are presented in table 6 (refs. [55-64]) ${ }^{*}$. Let us consider each in turn to understand the claims of $\rho / \omega$ dominance and dominance of certain helicity amplitudes using the notation $n_{1}, n_{2}$ for meson and baryon vertices respectively. Neutral photoproduction should be dominated by $\omega$ because the $\gamma_{\rho}$ coupling (vector dominance) and $\omega$ NN vertex are stronger than corresponding couplings with $\rho$-exchange. Thus we have $\left|n_{1}\right|=1$ and $n_{2}=0$ at meson and baryon vertices,

* A similar table has been independently prepared by Harari [55]. 
Table 6

Zero near $t \approx-0.6$ with vector-meson exchange.

\begin{tabular}{|c|c|c|c|c|c|c|c|}
\hline \multirow{2}{*}{ Reaction } & \multirow{2}{*}{$\begin{array}{l}\text { Dominant } \\
\text { exchange }\end{array}$} & \multicolumn{3}{|c|}{$\begin{array}{l}\text { Dominant } \\
\text { helicity }\end{array}$} & \multirow{2}{*}{$\begin{array}{c}\text { Dip } \\
\text { predicted } \\
\text { conventional } \\
\text { models } \\
\text { (N or V) }\end{array}$} & \multirow{2}{*}{$\begin{array}{l}\text { Dip } \\
\text { predicted } \\
\text { SCRAM }\end{array}$} & \multirow{2}{*}{$\begin{array}{c}\text { Dip } \\
\text { observed }\end{array}$} \\
\hline & & $\left|n_{1}\right|$ & $\mid n_{2}$ & $n=1 ?$ & & & \\
\hline$\gamma p \rightarrow \pi^{\circ} p$ & $\omega$ & 1 & 0 & yes & yes & yes & yes \\
\hline $\left.\begin{array}{l}\pi^{-} p \rightarrow \pi^{0} n \\
\pi^{+} p \rightarrow \pi^{\circ} \Delta^{++}\end{array}\right\}$ & $\rho$ & 0 & 1 & yes & yes & yes & yes \\
\hline $\left.\begin{array}{l}\pi^{-} p \rightarrow \omega n \\
\pi^{+} p \rightarrow \omega \Delta^{++}\end{array}\right\}$ & $\rho$ & 1 & 1 & no & yes & no & no? \\
\hline$\gamma p \rightarrow \eta p$ & $\rho$ & 1 & 1 & no & yes & no & no \\
\hline$\left(\gamma p \rightarrow \pi^{+} n\right)-\left(\gamma n \rightarrow \pi^{-} p\right)$ & $\rho$ & 1 & 1 & no & yes & no & no \\
\hline$(n p \rightarrow p n)-(p \bar{p} \rightarrow n \bar{n})$ & $\rho$ & 1 & 1 & no & yes & no & $?$ \\
\hline
\end{tabular}

respectively. $\mathrm{Pi}$ charge-exchange scattering must be $\rho$-exchange and so has $\left|n_{2}\right|=1$. The production of $\omega$ should be dominated by $\rho$-exchange at high enough energy. The $\omega$ is produced with helicity \pm 1 in $\rho$-exchange so $\left(\left|n_{1}\right|,\left|n_{2}\right|\right)=(1,1)$. Thus $(n, x)=(0,2)$ and $(2,0)$. Photoproduction of $\eta$ should be dominated by $\rho$-exchange rather than $\omega$-exchange because $g(\gamma \rho \eta) \approx$ $\approx g(\gamma \omega \eta)$ according to vector dominance and SU(3) arguments. The dominant $\rho$-exchange amplitudes are $\left|n_{1}\right|=\left|n_{2}\right|=1$, thus $(n, x)=(0,2)$ and $(2,0)$. The differential cross section differences are the products of the $\rho$-exchange amplitude with the $\pi$ plus $A_{2}$ amplitudes. The $\rho$-exchange should again be dominantly $\left|n_{1}\right|=\left|n_{2}\right|=1$.

In all these cases, in the conventional Regge models there should be a zero in the dominant amplitude and dip in the differential cross section at $\alpha=0$, near $t=-0.6$. In SCRAM there is a zero in the first three reactions near $t=-0.6$ because of $n=1$ pole-cut interference and no such zero in the other cases, because $n \neq 1$.

We also note that model $\mathrm{V}$ predicts an $(n, x)=(0,0)$ zero near $-t=0.6$. It is unobserved at this location. This case was discussed above in connection with structure near $-t=0.2$.

Experiment [56-64]: The zero in the vector-meson-exchange amplitude with helicity flip at either or both vertices is observed (i.e., as a differential cross section dip or sharp break) only in cases where helicity flip occurs at only one vertex, i.e., for $n=1$, as predicted in SCRAM. A dip or sharp break at $-t \approx 0.5$ in the differential cross section for $\gamma p \rightarrow \pi^{\circ} \mathrm{p}$ and a clear dip at $-t \approx 0.6$ in $\pi^{-} \mathrm{p} \rightarrow \pi^{\circ} \mathrm{n}$ are observed at high energies (in agreement with all models). For $\omega$-production, dominance of $\rho$ is not valid at $3.7 \mathrm{GeV} / c$ where the best data exists. No dip in the differential cross section is evident. This reaction is discussed further, below. Evidence that there is no dip or sharp break in $\gamma p \rightarrow \eta n$ and no zero for $d \sigma(p \bar{p} \rightarrow n \bar{n}) / d t$ $-\mathrm{d} \sigma(\mathrm{np} \rightarrow \mathrm{pn}) / \mathrm{d} t$ is fairly good but, perhaps, would not persuade everyone. The data on 


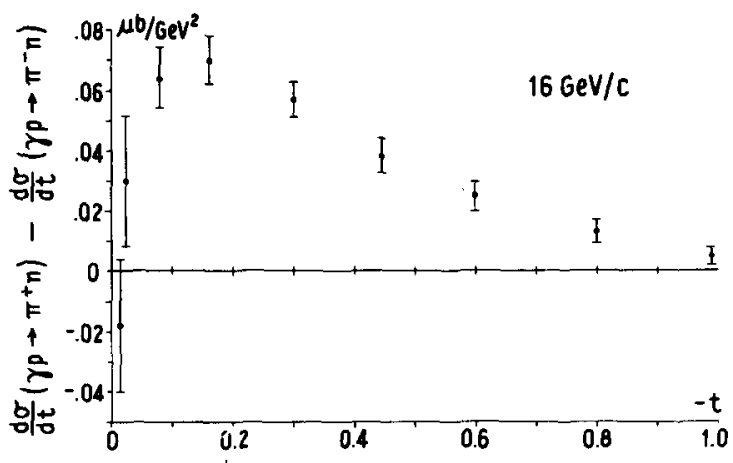

Fig. 6. The difference between $\pi^{+}$and $\pi^{-}$photoproduction cross sections, which is proportional to the $\rho$-exchange amplitude. All amplitudes have helicity flip at least at the $\gamma \rightarrow \pi$ vertex, and therefore all conventional models predict a zero near $-t=0.6$. SCRAM and the data have no zero.

$$
\frac{\mathrm{d} \sigma}{\mathrm{d} t}\left(\gamma \mathrm{p} \rightarrow \pi^{+} \mathrm{n}\right)-\frac{\mathrm{d} \sigma}{\mathrm{d} t}\left(\gamma \mathrm{n} \rightarrow \pi^{-} \mathrm{p}\right)=2 \operatorname{Re} A_{\rho}\left(A_{\pi}+A_{\mathrm{A}_{2}}\right)^{*},
$$

is at very high energy $(16 \mathrm{GeV} / c)$ so that the assumption of leading trajectory dominance is reasonable, and the data is good. The data is shown in fig. 6. This is very clear evidence for the lack of a nonsense wrong signature zero in the $\rho$-exchange amplitude. Note that absorptive cuts calculated with elastic intermediate state prescription would not remove the prediction of a change of sign in (37) if the Regge pole amplitude has a nonsense wrong signature zero. In this case the cut must simply move the zero in, because the cut, which is evaluated in integrating over intermediate angles, must be roughly out of phase with the phase of the pole term at small angles.

The reaction

$$
\pi^{+} \mathrm{p} \rightarrow \omega \Delta^{++},
$$

should be dominated by $\rho$-exchange at high enough energy. For $\rho$-exchange, dominance of $\left|n_{2}\right|=1$ (the baryon vertex) and $\left|n_{1}\right|=1$ (the meson vertex) are expected. However at the somewhat low experimental energy of $3.7 \mathrm{GeV} / c,\left|n_{1}\right|=\left|n_{2}\right|=1$ is not dominant (there may be large contributions from a $t$ channel double Reggeon cut, or exchange of a single Reggeon on a lower trajectory - these will be most difficult to distinguish; or it might be mis-identification of $\left.\Delta^{\prime} s\right)$. However selection of $\left|n_{1}\right|=1$ using the decay angular distribution of the $\omega$ should enhance $\rho$-dominance. One can examine the natural parity cross section

$$
\left(\rho_{11}+\rho_{1-1}\right) \frac{\mathrm{d} \sigma}{\mathrm{d} t},
$$

where the indices refer to the $s$-channel c.m. helicities. If the pole and cut amplitudes of SCRAM are combined (e.g., from fig. $5 \mathrm{c}$ and $d$ ) there is a zero in (38) in the neighbourhood of $-t=0.6$. Such a zero is also predicted with conventional Reggeons as a nonsense wrong signature point. Meanwhile, if 
either one of the terms in (38) is taken separately, SCRAM predicts no zero near $t=-0.6$, but the zero is predicted in the other models; the data shows no zero but is not conclusive. Higher energy, high statistics data is needed.

Conclusion: Experiments on zeros at $t \approx-0.6$ in the cases $n=1$ and $\left|n_{i}\right| \neq 0$ with vector meson exchange are scarce. The only powerfully persuasive measurement indicating that such a zero does not occur if $\left|n_{i}\right|=1$ but $n \neq 1$ is the difference between $\pi^{ \pm}$photoproduction. Regardless of cuts, this experiment indicates that the $\rho$-nonsense wrong-signature zero is not present in the pole amplitude. Most other experiments also favor the SCRAM model against the Regge pole models with nonsense wrong-signature zeros, although not so convincingly.

Structure at $t \approx 0 ; \pi$-exchange peaks

Theory: The pion pole, at $t=+0.02 \mathrm{GeV}^{2}$, is sufficiently near to the physical region to be detected when $\pi$-exchange is allowed by the quantum numbers. Thus it is of great interest to examine such cross sections. Complications from spin are very important, so the shapes of these cross sections vary considerably even in their qualitative features.

The cross section in all cases is a sum of squares of terms of the form

$$
\begin{array}{cc}
\frac{g g^{\prime} m_{\pi}^{2}}{t-m_{\pi}^{2}}+c_{00} & \text { for }(n, x)=(0,0), \\
\sqrt{-t}\left(\frac{g g^{\prime} m_{\pi}}{t-m_{\pi}^{2}}+c_{10}\right) & \text { for }(n, x)=(1,0), \\
-t\left(\frac{g g^{\prime}}{t-m_{\pi}^{2}}+c_{20}\right) & \text { for }(n, x)=(2,0), \\
\frac{-t g g^{\prime}}{t-m_{\pi}^{2}}+c_{02} & \text { for }(n, x)=(0,2) .
\end{array}
$$

The $c$ are slowly varying relative to the pole terms. They are usually of comparable magnitude to the pole term at $t \approx-m_{\pi}^{2}$. Thus all cross sections which include pion exchange should be studied for $\pi^{-} t \lesssim 0.02 \mathrm{GeV}^{2}$ by a parameterization such as

$$
\left(4 q^{2} s\right) \frac{\mathrm{d} \sigma}{\mathrm{d} t} \approx A+\frac{B m_{\pi}^{2}}{t-m_{\pi}^{2}}+\frac{G m_{\pi}^{4}}{\left(t-m_{\pi}^{2}\right)^{2}},
$$

(rather than by exponentials, as is a common partice). The constant coefficient $G$ of the $\left(t-m_{\pi}^{2}\right)^{-2}$ term can be expressed in terms of the pion couplings, which are often known or are something we want to extract from the data. The quantities $A$ and $B$ are coefficients which can depend on the details of non-pion background, as well as on the pion. They are, therefore, not determined without a detailed fit to the experimental data. 
The conventional evasive Regge-pole models provide quite distinctive predictions for many processes due to effects of spin, although, as is well known, these models are immediately contradicted by experimental data, it is useful to consider the structure of the evasive Regge poles since they provide the input to SCRAM.

The distinctive features of pion exchange arise because there is only one coupling of the pion at each of the experimentally accessible vertices. More couplings are prevented at the $\pi \mathrm{NN}$ vertex by conservation of $\mathrm{G}$ parity, at other vertices by "nonsense decoupling", i.e., by the low spin of the pion. (Its Regge recurrences do couple in more than one way.) The results, when expressed in terms of high-energy $s$-channel helicity flips $\left|n_{i}\right|$ at the vertex are

$$
\begin{aligned}
\left|n_{\mathrm{B} \rightarrow \mathrm{B}}\right| & =1, \\
\left|n_{\mathrm{B} \rightarrow \mathrm{B}^{*}}\right| & =0 \text { dominates, } \\
\left|n_{\mathrm{PS} \rightarrow \mathrm{V}, \mathrm{T}}\right| & =0 \text { dominates, } \\
\left|n_{\gamma \rightarrow \pi}\right| & =1,
\end{aligned}
$$

where $\mathrm{B}, \mathrm{B}^{*}$, PS, V, T stand for isospin $\frac{1}{2}$ baryon, isospin $\frac{3}{2}$ baryon, pseudoscalar meson, vector meson, and tensor meson, respectively. Each vertex contains a factor $(-t)^{\frac{1}{2}}\left|n_{i}\right|$, as discussed in subsect. 3.1. Thus, in $\gamma \mathrm{p} \rightarrow \pi^{+} \mathrm{n}$, for example, both vertices have $\left|n_{i}\right|=1$, and the evasive Regge pole amplitude vanishes like $t$ in the forward direction; $\pi \mathrm{N} \rightarrow \rho \Delta$, on the other hand, is dominated by $\left|n_{i}\right|=0$ at each vertex, and does not vanish. In an evasive Regge-pole model, all trajectories have the same behaviour near $t=0$ in each helicity amplitude. Therefore the part of the contributions of other poles which interferes with pion exchange has the same properties as the pion exchange itself. Only the incoherent part of the contributions of other poles is different, and it contributes only to the $A$-term in eq. (36b).

The conspiring Regge-pole model (see, for example, the review article by Hite [33]) has also been applied to pion exchange reactions. In this model, for the cases we are considering, the vertex has a factor $(-t)^{\frac{1}{2}\left(1-\left\lceil n_{i}\right\rceil\right)}$, determined by factorization. (General formulas are in the footnote p. 290). For the $n=2$ amplitude, this factor leads to no vanishing, while kinematics requires a vanishing like $t$. For this reason, a conspiring Regge trajectory, with opposite parity, must just cancel the contribution to the $n=2$ amplitude of the trajectory under consideration. In the conspiracy model for the pion $\gamma \mathrm{p} \rightarrow \pi^{+} \mathrm{n}$ will not vanish at $t=0$, while the dominant part of $\pi \mathrm{N} \rightarrow \rho \Delta$, with $\left|n_{i}\right|=0$ at each vertex, will vanish [65].

Regge cuts, generated by absorption, can change the behaviour of the pure pole models *. The cuts do not correspond to a single parity, nor do

* A Regge-cut model was introduced by Frøyland and Gordon [66a], see also Amati et al. [66b]. Absorptive cuts with $\pi$-exchange have been considered by Kaidalov and Karnakhov and by Benfatto et al. [66c]. For absorption corrections to non-Reggeized to exchange see refs. $[23,66 d]$. 
they factor as poles do, and parity and factorization are the principle causes of the Regge-pole structure near $t=0$. It turns out, as expected, that the absorption model gives futs which vanish only as strongly as is kinematically required, i.e. as $(-t)^{\frac{1}{2}} n$. Rough estimates of the cut contribution can easily be made. We assume evasive input Regge-pole amplitudes, to avoid the necessity of the ad hoc introduction of a conspiring trajectory of positive (natural) parity. The $x=0$ amplitudes are all concentrated at small $-t$ (after removal of the kinematic factor $(-t)^{\frac{1}{2} n}$ ) because of the $\left(t-m_{\pi}^{2}\right)^{-1}$ factor. Thus in impact parameter space, they are spread over large areas, mostly beyond the range of absorption. Therefore the cuts are very small in the $(n, x)=$ $(0,0),(1,0)$ and $(2,0)$ amplitudes. The $(n, x)=(0,2)$ amplitude, however, involves a factor $t /\left(t-m_{\pi}^{2}\right)=1+m_{\pi}^{2} /\left(t-m_{\pi}^{2}\right)$. As before, the $m_{\pi}^{2} /\left(t-m_{\pi}^{2}\right)$ term is very slightly absorbed. The 1 term however, is significantly absorbed. The amount of absorption (near $t=0$ ) is approximately the product of:

(i) $\lambda$, which measures the strength of coherent inelastic effects, (ii) $T_{\mathrm{el}}(b=0)=\sigma_{\text {tot }} /\left(4 \pi \times\right.$ slope of $\left.\mathrm{d} \sigma_{\mathrm{el}} / \mathrm{d} t\right) \approx \frac{2}{3}$ for meson-baryon reactions, (iii) a factor of about $\frac{1}{2}$, arising from the fact that the range of a Regge pole effect is comparable to the range of absorption, and (iv) the size of the Regge pole (with $t /\left(t-m_{\pi}^{2}\right)$ replaced by unity). All these factors appear in eq. (A.12a) of HKPR [1]. Putting them together we get, for meson-baryon processes,

$$
\operatorname{cut}_{(0,2)} \approx \frac{1}{3} \lambda \times \text { pole }_{(0,2)},
$$

and slightly larger for baryon-baryon processes.

There is one remaining model which has been applied to photoproduction, namely, the fixed pole model [67]. The usual proof that fixed $j$-plane poles are not allowed in determining high energy behaviour does not apply to photoproduction, since the proof involves non-linear unitarity, not possessed by photoproduction to order $e$. This has led to models involving fixed poles. For example, the observation that the $s$-channel nucleon Born term approximately fits the near-forward $r p \rightarrow \pi^{+} n$ data, if taken seriously, is described as fixed poles in the $j$-plane [68] ${ }^{*}$. (If this observation is interpreted in terms of duality ideas, however, it can correspond to any $j$-plane singularity near $j=0$, in particular, to Regge cuts.) There are, however, strong theor etical arguments to rule out fixed poles $[69,70]$.

Although these theoretical arguments rest on specific dynamical models, the result is suggested by quite general considerations. The pion pole occurs in photoproduction. Since the pion is composite, when taken off mass shell, it is a moving pole following the trajectory $\alpha_{\pi}(t)$. The proposed fixed poles however involve the pion pole (possibly indirectly, in terms of the nucleon pole, which contains the pion pole by gauge invariance); this fixed pole im-

* The Feynman graph with a nucleon pole at $s=M^{2}$ appears to be an appropriate addition to the $\pi$-exchange Feynman graph in order to preserve gauge invariance. However, only the singularity is required. The high-energy tail of the nucleon term can be very different. The absorptive and other models discussed here are gauge invariant (See ref. [73] for an extensive discussion of gauge invariance). 
plies an elementary pion. The usual theoretical arguments $[69,70]$ against fixed poles rest on the compositeness of the external pion. Gauge invariance, however, intimately relates the properties of the external and the internal pions.

Experiment: The experiments conveniently group themselves according to the helicity amplitudes which dominate $\pi$-exchange:

$$
\begin{aligned}
& (n, x)=(0,0): \pi^{+} \mathrm{p} \rightarrow \rho^{0} \Delta^{++}, \quad \mathrm{K}^{+} \mathrm{p} \rightarrow \mathrm{K}^{* 0} \Delta^{++}, \\
& (n, x)=(0,2) \text { and }(2,0): \gamma p \rightarrow \pi^{+} \mathrm{n}\left(\text { ref. [62]), } \gamma \mathrm{n} \rightarrow \pi^{-} \mathrm{p}\right. \text { (ref. [62]), } \\
& \mathrm{np} \rightarrow \mathrm{pn}(\text { ref. [63]), } \mathrm{p} \overline{\mathrm{p}} \rightarrow \mathrm{n} \overline{\mathbf{n}} \text { (ref. [64]), } \\
& (n, x)=(1,0): \mathrm{pp} \rightarrow \mathrm{n} \Delta^{++}\left(\text { ref. [71]), } \quad \pi \mathrm { N } \rightarrow \rho \mathrm { N } \left(\text {ref. [72]), } \quad \mathrm{KN} \rightarrow \mathrm{K}^{*} \mathrm{~N} .\right.\right.
\end{aligned}
$$

We have associated reactions for which there is extensive data with the helicities of the dominant helicity amplitudes for $\pi$-exchange. This is not to suggest that $\pi$-exchange completely dominates these reactions. There are many cases where other exchanges are very important. However, we are looking for structure at very small $-t$, i.e., $-t \lesssim 0.02$ to 0.04 , which will be due to $\pi$-exchange. Such structure is easily seen in many, but not all, of the above reactions. Furthermore, the energy dependence near $t=0$ is consistent with $\pi$-exchange; however the energy dependence does not provide a sensitive test against important contributions due to exchange of other Reggeons.

The above association is determined by the properties of the $\pi$-exchange vertices, e.g., as given by eq. (36c). The experimental differential cross sections show the following principal features: The $(n, x)=(0,0)$ cases show a large sharp peak naturally associated with the pion for $-t \lesssim 0.1$. The $(n, x)=(0,2)$ and $(2,0)$ cases show a smaller sharp forward spike contained within $-t \approx 0.02$, followed by a shoulder out to $-t \approx 0.1$, except for $\bar{p} \overline{\mathbf{p}} \rightarrow \mathrm{n} \overline{\mathbf{n}}$ where the cross section is very much larger than in the crossed reaction $\mathrm{np} \rightarrow \mathrm{pn}$, suggesting that $\pi$-exchange features may be hidden. The $(n, x)=(1,0)$ cases do not exhibit obvious structure in the near forward direction. All these cross sections fall rather smoothly like $\mathrm{e}^{a t}$ with $a \approx 5$ to $10 \mathrm{GeV}^{-2}$ aside from the structure mentioned. It will be seen below that certain important but less prominent features of these cross sections have probably not been seen because of difficulties of measurement.

The reactions associated with $(n, x)=(0,2)$ and $(2,0)$ are the key to the behaviour of $\pi$-exchange near $t=0$. We can immediately dispose of a theoretical description which involves "evasive poles" only, since several reactions show $\pi$-exchange peaks associated with $(n, x)=(0,2)$ instead of dipping in the forward direction (note, for $\pi$-photoproduction there is no $(n, x)=(0,0)$ amplitude so the observed peak rules out this description), or with "conspiring poles" only, where comparison of related cross sections, particularly $\pi^{+} p \rightarrow \rho^{\circ} \Delta^{++}$, using factorization, fails [65]. These reactions can be fit using a model with both evasive and conspiring trajectories. No less than 
three trajectories associated with the $\pi$ are needed: $\pi, \pi^{\prime}, \pi^{\prime \prime}$. With these exchanges, and with the contributions due to exchange of $\rho$ and A noted above, a fit to data in the $-t \approx 0$ region, similar to that obtained in the strong absorption model below, can, perhaps, be obtained. (Note that experiment rules out a conspiracy model for $\mathrm{K}$-photoproduction parallel to that for $\pi$ (ref. [73]).) No particles have been observed in association with $\pi^{\prime}$ and $\pi$ ". These facts, and its artificiality, render this approach quite unsatisfactory. Another theoretical model involves the addition of fixed poles at $\alpha=0$ (i.e., poles in the $t$-channel angular-momentum plane) to conspiring and evasive poles. These fixed poles are only considered in photoproduction. One of the most unpalatable features of such fixed pole contributions is the need to recreate similar features of the data for $(n, x)=(0,2)$ in $n p \rightarrow$ pn by a wholly different mechanism, such as conspiracy. Another hadronic process $\pi^{-} \mathrm{p} \rightarrow \rho^{\mathrm{o}} \mathrm{n}$ (ref. [72]) has, in all probability, the same forward peak, when the $\rho$ has helicity \pm 1 , as is observed in photoproduction, and so would more naturally be explained in the same way as photoproduction. If there are fixed poles backward photoproduction would be expected to have a fixed pole at $\alpha=\frac{1}{2}$ in disagreement with the observed energy dependence [70].

The absorption approach with evasive poles is much more promising because the $(n, x)=(0,2)$ amplitudes should appear as in fig. $5 \mathrm{~d}$. The data for these reactions (except $\mathrm{p} \overline{\mathrm{p}} \rightarrow \mathrm{n} \overline{\mathrm{n}}$ ) where $\pi$ exchange is dominated by $(n, x)=$ $=(0,2)$ and $(2,0)$, indeed looks roughly like the sum of such an $(n, x)=(0,2)$ amplitude and a $(2,0)$ amplitude (fig. $5 \mathrm{c}$ ) which peaks at relatively small $-t$, near 0.06 because it is $\pi$-exchange. This $(n, x)=(2,0) \pi$-exchange contribution is directly visible as a shoulder in certain $n p \rightarrow$ pn and $\gamma p \rightarrow \pi^{+} n$ angular distributions.

Let us investigate these cases more quantitatively. We have $(n, x)=(0,0)$, $(1,0),(2,0),(0,2)$ amplitudes; they have the dimensionless forms given in eq. (36a). The quantity $C_{02}$ in that equation contributes in the forward direction. The "principal cut" associated with $\pi$-exchange can be estimated using eq. (36r)

with

$$
C_{02}=C_{02 \pi}+C_{02}^{\prime}
$$

$$
C_{02 \pi} \approx-\frac{1}{3} \lambda g g^{\prime},
$$

where $\lambda=1$ corresponds to the elastic absorption prescription and where, $C_{02}^{\prime}$ contains contributions due to (absorption corrections to) exchange of other Reggeons. In terms of the quantity $G$ of eq. (36b) we have

$$
G=2\left(g g^{\prime}\right)^{2}=e^{2} g_{\pi}^{2} / 4 \pi=0.53 \mathrm{mb} \mathrm{GeV}^{2},
$$

for $\gamma p \rightarrow \pi^{+} \mathrm{n}$ and $\gamma \mathrm{n} \rightarrow \pi^{-} \mathrm{p}$ and

$$
G=g_{\pi}^{4} / 4 \pi=1.06 \mathrm{GeV}^{2} \cdot \mathrm{b},
$$

for $\mathrm{np} \rightarrow \mathrm{pn}$, where $e^{2} / 4 \pi=1 / 137$ and $g_{\pi}^{2} / 4 \pi=14.7$. We wish to compare 
experiments and theory in the forward direction. Theoretically

$$
4 q^{2} s \frac{\mathrm{d} \sigma}{\mathrm{d} t} \underset{t \rightarrow 0}{=}\left|C_{02}\right|^{2} \approx \frac{1}{2}\left|\frac{1}{3} \lambda+C\right|^{2} \frac{1}{2} G .
$$

Experiments averaged for $\gamma \mathrm{p} \rightarrow \pi^{+} \mathrm{n}$ and $\gamma \mathrm{n} \rightarrow \pi^{-} \mathrm{p}$ (ref. [62]) for $P_{1 \mathrm{lab}}$ $\geqslant 5 \mathrm{GeV} / c$ yield

$$
4 q^{2} s \frac{\mathrm{d} \sigma}{\mathrm{d} t}=0.6 G( \pm 15 \%),
$$

while the old $\mathrm{np} \rightarrow$ pn experiment at $8 \mathrm{GeV}$ yields $0.23 \mathrm{G}$ and a new experiment at a variety of energies has roughly twice the absolute cross section (preliminary results) [63]. It appears for $\mathrm{np} \rightarrow \mathrm{pn}$

$$
4 q^{2} s \frac{\mathrm{d} \sigma}{\mathrm{d} t}=(0.4 \text { to } 0.5) G \text {. }
$$

It is straightforward to describe the above experiments by choosing $\lambda \approx 2$ to 3 , and including significant but not dominant $\rho$ - and A-contributions near $t=0$. No one has yet found a satisfactory way to describe these experiments with $\lambda=1$, with most of the contribution due to the other exchanges (the known behaviour in phase and in charge dependence for $\rho$ - and A-exchange contributions restricts one's freedom). We conclude that while the elastic absorption prescription is quantitatively inadequate, the absorption model, with strong-absorption, can be satisfactory for these reactions. Indeed a detailed and satisfactory fit to these four reactions and related processes has been recently carried out [30].

It remains to briefly describe the other two sets of reactions in the absorption model. Consider those associated with $(n, x)=(0,0) \pi$-exchange. It is hard to make predictions or extract numbers for $A$ and $B$ terms (eq. (36b)) in the differential cross section. It is difficult to normalize the data with two broad resonances present and it is difficult to compare experiment and theory at moderate energies because of the large $t_{\min }$ which changes from event to event. The data is consistent with a relatively steep differential cross section. As discussed above, the amount of absorption is small even in strong absorption models.

The reactions associated with $(n, x)=(1,0) \pi$-exchange should on very close examination show a small $(n, x)=(0,2) \pi$-exchange contribution with its characteristic shape. This is discussed further in the next section of this paper. This has not been seen. Even the turnover near $t=0$ associated with large $(n, x)=(1,0)$ does not show up strongly. The data is, however, certainly consistent with the strong absorption model with evasive poles.

Conclusion: The pure evasive Regge-pole model is ruled out by the existence of a forward peak in $\gamma p \rightarrow \pi^{+} n$, and the pure conspiracy model is ruled out by $\pi^{+} p \rightarrow \rho^{\circ} \Delta^{++}$. Thus, a pure pole model requires three trajectories with intercept near $j=0$. Of these, only the pion has been seen as a particle. Therefore, pure pole models are very unlikely to be valid.

The fixed pole model is unpalatable on theoretical grounds. Moreover, it makes no attempt to explain purely hadronic processes. In addition, there is no fixed pole at $j=\frac{1}{2}$ in backward photoproduction, as would be expected. 
If the data is to be explained by cuts, they must be very strong. The observed cross sections are about an order of magnitude larger than those obtained with $\lambda=1$. In fact, the necessary strength of the cut is even larger than SCRAM would give with the same $\lambda$-value as in other processes.

Summary: All the various aspects of the angular structure favour SCRAM over the conventional models. The observed structure depends primarily on $(n, x)$, rather than the details of the exchange. Dips occur at $-t=0.2$ and 0.6 for $(n, x)=(0,0)$ and $(1,0)$ respectively. This is the position of the first diffraction minimum of a ring source with a radius of one $\mathrm{fm}$, and an azimuthal dependence $e^{i n \varphi}$.

On the other hand, the conventional models do not correctly explain the angular structure; $\pi$-exchange at $t=0$ requires either strong cuts or unappealing assumptions. The dip structure at 0.2 and 0.6 in conventional models is attributed to nonsense wrong-signature zeros and accidental dynamical zeros. These assumptions can be made to work in individual processes.

However, the conventional models require factorization of these zeros. The observed structure does not factorize. Thus, the conventional models force one to have too many zeros in some amplitudes, or not enough in others. As examples, the absence of a general dip at $u \approx-0.2$ for $\mathrm{N}$-exchange and the absence of a zero at $t \approx-0.6$ in $\mathrm{d} \sigma\left(\gamma \mathrm{p} \rightarrow \pi^{+} \mathrm{n}\right) / \mathrm{d} t-\mathrm{d} \sigma\left(\gamma \mathrm{m} \rightarrow \pi^{-} \mathrm{p}\right) / \mathrm{d} t$ argue strongly against nonsense wrong-signature zeros.

The conventional model, which we call the N-model, disagrees qualitatively with experiment. The exchange degenerate model, which we call the $\mathrm{V}$-model, also disagrees with experiment. However, if the V-model is considered only as a very rough approximation, it does have features in agreement with the experimental data and with SCRAM. It has a linear zero in each vector exchange amplitude. (Vector exchange is most easily studied experimentally.) Only the positions of the zeros are incorrect for agreement with experiment (ignoring in this approximation the difficulties with factorization of zeros). We remark that absorptive cuts applied to the $\mathrm{V}$-model are not sufficient to position the zeros correctly.

\subsection{Experimental predictions of SCRAM}

One can say a great deal about the general structure of amplitudes and of many reactions in SCRAM, independently of the details of the parameters. (Note: SCRAM is defined in subsect. 3.4.) Some predictions are contained in table 6.

All amplitudes are classified according to $s$-channel helicities and the net helicity flip $n=\left|\lambda_{\mathrm{a}}-\lambda_{\mathrm{b}}-\lambda_{\mathrm{c}}+\lambda_{\mathrm{d}}\right|$ and $x=\left|\lambda_{\mathrm{a}}-\lambda_{\mathrm{c}}\right|+\left|\lambda_{\mathrm{b}}-\lambda_{\mathrm{d}}\right|-n$.

Apart from the pion exchange, which can be understood in the same terms as the following but which gives somewhat different numerical values because of the importance of the nearby pole, the model has the properties:

(a) For any exchange for any process (e.g., $\rho, A_{2}, N, \Delta$, etc.) an amplitude with $(n, x)=(0,0)((n, x)=(1,0))$ has pole-cut destructive interference about $0.2(\mathrm{GeV} / c)^{2}\left(0.6(\mathrm{GeV} / c)^{2}\right)$ away from the forward or backward direction. The uncertainty in parameters can shift these points by perhaps $25 \%$ from process to process.

(b) All helicity amplitudes vanish at the forward direction as $\left(t_{\mathrm{o}}-t\right)^{\frac{1}{2} n}$; 
thus the behaviour near $t=t_{\mathrm{o}}$ and the point of the pole-cut interference are closely related by the dependence on $n$. Similar remarks hold for the backward direction here and below.

(c) The pole-cut interference near $t=-0.2$ tends to give a significant dip in the magnitude of the amplitude since the real and imaginary parts of the amplitude vanish at points that are not far separated. By $t=-0.6$ the relative phase of pole and cut is far enough from $\pi$ that one can get a shallow dip or just a break, where the cut with its flatter slope takes over.

(d) Differential cross sections are incoherent sums of the absolute squares of helicity amplitudes. The $n=1$ amplitudes, which vanish at $t=t_{0}$, have a peak near $t=-0.2$, just where the $n=0$ amplitudes have a dip. Thus if the two contribute to some reaction in about equal amounts (as in $\pi^{-} p \rightarrow p \pi^{-}$) one will see no dips. In general one must have information on couplings to know which amplitudes dominate.

Using these results, let us give some qualitative predictions for angular distributions.

(i) $\mathrm{d} \sigma / \mathrm{d} t$ or $\mathrm{d} \sigma / \mathrm{d} u$ for any process with high-spin external particles, (e.g., $\pi \mathrm{N} \rightarrow \mathrm{f}^{0} \mathrm{~N}, \pi \mathrm{N} \rightarrow \rho \Delta, \mathrm{pp} \rightarrow \pi \mathrm{d}, \pi \mathrm{N} \rightarrow \mathrm{N} \rho$, etc.) will be the sum of squares of many terms and will not show dips away from the forward direction unless the couplings are very special. (Some models, of course, have particular couplings which result in vanishing of certain helicity amplitudes.)

(ii) $\mathrm{d} \sigma / \mathrm{d} t(\mathrm{~d} \sigma / \mathrm{d} u$ ) for all processes, except for scattering of spinless particles on spinless particles, will show a break in slope for $-t(-u) \gtrsim 0.6$ because the $n=1$ cut with its shallower slope is beginning to dominate there and, in addition, the $n=0$ amplitude has a secondary maximum there. The $n>1$ amplitudes are not dominant except, in principle, for very high external spins.

(iii) The $n=0$ amplitude for $\Delta$-exchange in $\pi^{-} \mathrm{p} \rightarrow \mathrm{p} \pi^{-}$will have a zero (and in particular its imaginary part vanishes) somewhere near $-u=0.2$, because all $n=0$ amplitudes do; as far as we know this prediction is unique to SCRAM. It can be tested, in principle and perhaps in practice, by evaluation of appropriate finite energy sum rules [74-77]. Similarly, the $n=1$ amplitude in $\pi^{+} \mathrm{p} \rightarrow \mathrm{p} \pi^{+}$in SCRAM does not have a zero near $u=-0.2$, while in models with nonsense wrong-signature zeros it does vanish there. Again, finite energy sum rules might be able to distinguish between these alternatives.

Similar effects hold for backward $\mathrm{K}^{+} \mathrm{p} \rightarrow \mathrm{pK}^{+}$; in SCRAM the $n=0$ amplitude will have a zero near $u=-0.2$, while in models with nonsense wrongsignature zeros or elastic absorptive cuts it will not.

(iv) The correlation between the forward behaviour and the dip structure implies for any differential cross section:

Significant dip at $-t(-u) \approx 0.6 \rightarrow$ forward (backward) turnover, $\rightarrow$ no significant dip at $-t(-u) \approx 0.2$.

Significant dip at $-t(-u) \approx 0.2 \rightarrow$ no forward (backward) turnover, $\rightarrow$ no significant dip at $-t(-u) \approx 0.6$. Note that these predictions cannot be reversed, i.e., the right hand statements do not imply the dips at left.

(v) Consider pion exchange in vector-meson production. A number of 
processes are discussed in previous sections. Here we want to make predictions for small $t$ for $\pi^{-} \mathrm{p} \rightarrow \rho^{\mathrm{O}} \mathrm{n}$ (ref. [72]) and $\mathrm{K}^{-} \mathrm{p} \rightarrow \overline{\mathrm{K}}^{* \mathrm{O}} \mathrm{n}, \mathrm{K}^{+} \mathrm{n} \rightarrow \mathrm{K}^{*} \mathrm{p}$. Labelling the c.m. helicity amplitudes with a superscript for the $\rho$ or $\mathrm{K}^{*}$ helicity and subscripts for the final and initial baryon helicity respectively, $M_{\lambda}^{\prime}, \lambda$, the analysis goes as follows [78]:

There are six independent helicity amplitudes which can be chosen as $M_{++}^{\mathrm{o}}, M_{+-}^{\mathrm{o}}, M_{++}^{+}, M_{--}^{+}, M_{+-}^{+}, M_{-+}^{+}$. Their $n$-values are respectively $(n, x)=$ $=(0,0),(1,0),(1,0),(1,0),(0,2)$, and $(2,0)$. We consider here only pion exchange, so to assure dominance of $\pi$-exchange dependence consider only $-t \lesssim 0.02$. At high energies the pion-exchange amplitudes with nucleon helicity flipped are a power of $s$ higher than those with nucleon helicity unchanged, so we drop the three amplitudes with $\lambda^{\prime}=\lambda$.

We then have to consider $M_{+-}^{0}, M_{+-}^{+}$and $M_{-1}^{+}$. Near the forward direction the pole contributions go in general as $\left(t_{0}-t\right)^{\frac{1}{2}(n+x)}$. Parity conservation at the vertices gives for the poles (but not for the full amplitudes) $M_{+-}^{+}=M_{-+}^{+}$. Because these have different $n$, they are absorbed differently and are not expected to be equal experimentally.

Because $M_{+-}^{+}$has a pole part equal to the pole of $M_{-+}^{+}$, which is proportional to $t, M_{+-}^{+}($with $(n, x)=(0,2))$ consists of a pole which evades, i.e., which is of the form $t /\left(t-m_{\pi}^{2}\right)$, minus a large cut which does not vanish in the forward direction; $M_{-+}^{+}$(with $\left.(n, x)=(2,0)\right)$ behaves as $t /\left(t-m_{\pi}^{2}\right)$ with a small cut. Meanwhile, $M_{+-}^{0^{+}}$behaves as $(-t)^{\frac{1}{2}} /\left(t-m_{\pi}^{2}\right)$.

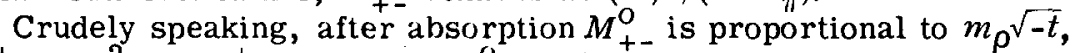
$M_{+-}^{+} \approx m_{\pi}^{2}$ and $M_{-+}^{+} \approx \ell$. Thus $M_{+-}^{0}$ is the dominant amplitude, while $M_{+-}^{+}$has a sharp peak as illustrated in fig. $5 \mathrm{~d}$ with the dip at $-t \approx 0.02$ and $M_{-+}^{+}$vanishes as $t$.

On the basis of these arguments we can make various predictions [78] for $-t \lesssim 0.02$. We denote c.m. density matrix elements by $\widetilde{\rho}$ and spin density matrix elements in the vector meson rest frame by $\rho$. These predictions are:

(1) $\rho_{11}$ and $\widetilde{\rho}_{11} \mathrm{d \sigma} / \mathrm{d} t$ have forward peaks

(2) $\rho_{00}$ and $\mathrm{d} \sigma / \mathrm{d} t$ have forward dips

(3) $\rho_{11} \mathrm{~d} \sigma / \mathrm{d} t$ is flat

(4) $\tilde{\rho}_{1-1} / \rho_{11}$ behaves like $-t / m_{\pi}^{2}$ for $-t \lesssim m_{\pi}^{2}$, reaching a value near +1 at $t \approx-m_{\pi}^{2}$. Since this quantity is equivalent to the photon asymmetry in charged photoproduction with polarized photons, we expect to find the vector dominance prediction well satisfied near $-t=m_{\pi}^{2}$, where the photon asymmetry is measured to be about 1 .

(vi) Consider the set of backward reactions that can only have $I=\frac{1}{2}$ exchange, e.g., $K^{-} p \rightarrow \Lambda \pi, \pi^{-} p \rightarrow \eta n$, etc. Since we know that $\pi^{+} p \rightarrow p \pi^{+}$is dominated by nucleon exchange and shows a deep dip near $-u=0.15$, in SCRAM we can conclude that the dominant helicity amplitude has $n=x=0$. If the $\mathrm{N}-\Lambda$ mass difference and $\eta-\eta^{\prime}$ mixing effects are unimportant, and if $\mathrm{SU}(3)$ can be assumed with the same $\sqrt{u}$ dependence for the F- and D-type couplings, then the helicity dependence of $\eta \mathrm{NN}$ and $\mathrm{KN} \Lambda$ will be the same as $\pi \mathrm{NN}$. In this case the $(n, x)=(0,0)$ amplitudes will dominate here too and give a deep dip.

(vii) If the $\omega \mathrm{NN}$ and $\rho \mathrm{NN}$ couplings were known we could predict the cross 
section for the process $K_{2} p \rightarrow K_{1} p$ (ref. [79]). Although the $\rho N N$ vertices can indeed be rather well determined from study of other reactions, the $\omega$-couplings obtained from $\gamma \mathrm{p} \rightarrow \pi^{\mathrm{o}} \mathrm{p}$ and $\gamma \mathrm{p} \rightarrow \eta \mathrm{p}$ unfortunately are not as well determined by present fits. If the $\omega N N$ charge coupling dominates as in the traditional lore, then the $n=0$ amplitude for $\mathrm{K}_{2} \mathrm{p} \rightarrow \mathrm{K}_{1} \mathrm{p}$ dominates and one expects a dip in toward $-t \approx 0.2$, and no forward turnover; while if the tensor coupling is not small the $n=1$ amplitude is not unimportant and there will be a forward flattening and a shallow dip or break moved outward toward $-t \approx 0.6$. In any case, $\mathrm{K}_{2} \mathrm{p} \rightarrow \mathrm{K}_{1} \mathrm{p}$ and $\gamma \mathrm{p} \rightarrow \pi^{\circ} \mathrm{p}$ and $\gamma \mathrm{p} \rightarrow \eta \mathrm{p}$ are closely related and taken together will fix the $\omega$ NN couplings.

\section{ENERGY DEPENDENCE OF R-TYPE REACTIONS IN SCRAM}

\subsection{General results}

In SCRAM a number of qualitative and semi-quantitative statements can be made without detailed parameter dependent calculations about the energy dependence of $\mathrm{R}$-type or single Reggeon exchange processes including strong diffractive cuts. It is common [80] to describe the $s$-dependence with the function $\alpha_{\text {eff }}(t)$

$$
\frac{\mathrm{d} \sigma}{\mathrm{d} t} \approx f(t) s^{2 \alpha_{\mathrm{eff}}(t)-2}
$$

where the fitting to $\alpha_{\text {eff }}$ is made, at present, in the region roughly $5-20 \mathrm{GeV} / c$.

It must be emphasized that the use of $\alpha_{\text {eff }}(t)$ is beset with snares. The actual cross section does not have an energy dependence $s^{2} \alpha_{\text {eff }}^{-2}$; i.e., the left side of eq. (39) does not depend on $s$ as indicated by the right side. A reasonable definition of $\alpha_{\text {eff }}$ should require that if a theory agrees with experimental data that the theoretical and experimental $\alpha_{\text {eff }}(t)$ agree. We thus determine $\alpha_{\text {eff }}(t)$ from a theoretical cross section by making a best fit to (39) with use of the experimental errors, i.e., one minimizes

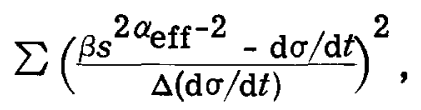

where $\Delta(\mathrm{d} \sigma / \mathrm{d} t)$ are the experimental errors, whether $\mathrm{d} \sigma / \mathrm{d} t$ is the theoretical or experimental cross section. It must be kept in mind that in both experimental and theoretical determinations, this procedure weights low energies (because the data is more accurate there) which is unfortunate (because our theories are less accurate there). Other methods of determining $\alpha_{\text {eff }}$ will weight theory at high energy and experiment at low energy; lack of agreement is not surprising.

In general each helicity amplitude is the sum over the contributions due to different allowed Reggeons, each contribution being the sum of pole and cut. The pole and cut are roughly $\pi$ out of phase at small $-t$, but may become relatively imaginary by $-t \approx 1$. Consider the cut due to compounding Reggeon exchange, with an energy dependence $s \alpha_{\mathrm{R}}(t)$, and diffraction with 
an energy dependence $s^{\alpha}{ }^{(t)}$. At given intermediate momentum transfers $t_{1}, t_{2}$, the energy dependence is given by $s\left[\alpha_{\mathrm{R}}\left(t_{1}\right)+\alpha_{\mathrm{V}}\left(t_{2}\right)-1\right]$. Let $t_{\mathrm{R}}$ and $t_{\mathrm{V}}$ be the kinematically allowed values of $t_{1}$ and $t_{2}$ which maximize the power of $s$. Then as $s \rightarrow \infty$ the energy dependence of the cut is given by [81]

$$
M^{\mathrm{C}} \leqslant \text { const. } \times \frac{{ }^{\left[\alpha_{\mathrm{R}}\left(t_{\mathrm{R}}\right)+\alpha_{\mathrm{V}}\left(t_{\mathrm{V}}\right)-1\right]}}{\ln s} .
$$

For linear trajectories,

$$
\begin{aligned}
& \sqrt{-t_{\mathrm{R}}}=\frac{\alpha_{\mathrm{V}}^{\prime}}{\alpha_{\mathrm{V}}^{\prime}+\alpha_{\mathrm{R}}^{\prime}} \sqrt{-t}, \\
& \sqrt{-t_{\mathrm{V}}}=\frac{\alpha_{\mathrm{R}}^{\prime}}{\alpha_{\mathrm{V}}^{\prime}+\alpha_{\mathrm{R}}^{\prime}} \sqrt{-t .}
\end{aligned}
$$

At current energies the distribution in angles will be more broadly spread, e.g., because the two scatterings will not be in the same plane. Thus the average values of $-t_{1},-t_{2}$ are larger than (41). Another reason why (40) is not quite achieved is that the cut discontinuity vanishes at the branch point, i.e., the double scattering does not occur at angles which exactly maximize the $s$-dependence. As a result of these arguments and the $\ln s$ in (40) it is seen that the cut branch point $\left[\alpha_{\mathrm{V}}\left(t_{\mathrm{V}}\right)+\alpha_{\mathrm{R}}\left(t_{\mathrm{R}}\right)-1\right]$ as a function of $t$ lies above $\alpha_{\text {eff }}(t)$ of the cut and $\alpha_{\text {eff }}^{\prime} \equiv \mathrm{d} \alpha_{\text {eff }}(t) / \mathrm{d} t$ is positive even if $\alpha_{\mathrm{V}}=$ const. From these double scattering arguments we see that at small $-t$, $\alpha_{\text {eff }}$ of the cut lies below $\alpha_{\text {pole }}$ while for large $-t, \alpha_{\text {eff }}$ of the cut lies above $\alpha_{\text {pole. }}$ Define $t^{\prime}$ to be the value of $t$ at which this transition occurs. It turns out that $-t^{\prime} \approx 0.1$ to 0.3 , increasing with increasing $n$ and $x$.

The $\alpha_{\text {eff }}$ for one helicity amplitude for Reggeon exchange (pole + cut) thus depends on whether $t \lessgtr t$, and whether $\mid$ pole $|\lessgtr|$ cut $\mid$. The two most important cases are sketched in fig. 7: for $x=0$ (non-evasive) with $-t$ at the dip (where $\mid$ cut $\mid$ begins to dominate $\mid$ pole $\mid$ ) greater than $-t^{\prime}$, and for $n=0$, $x=2$ (evasive) with $\mid$ cut $|>|$ pole $\mid$ throughout. For both curves $\alpha_{V} \approx 1$.

(a)

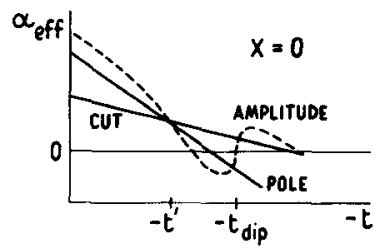

(b)

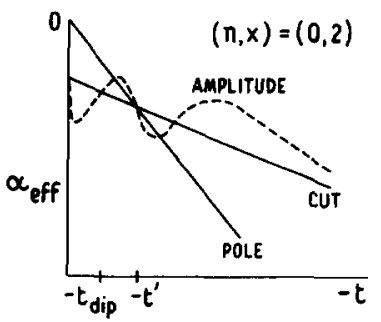

Fig. 7. Energy dependence in SCRAM of cross sections due to definite helicity flip $(n, x)$. In (a), for $x=0$ (and any $n$ ), the pole dominates at small angles (inside $-t_{\text {dip) }}$ and the cut at larger angles. At $t^{\prime}, \alpha$ of the pole equals the effective $\alpha$ of the cut. In (b), $(n, x)=(0,0)$ so that the cut dominates for $t$ inside $t_{\text {dip }}$, then the pole dominates, then at large $-t$ the cut dominates again. The rough effect of this complicated behavior is that $\alpha$ does not change rapidly. 
The qualitative predictions are as follows:

$$
\alpha_{\text {eff }}^{\prime}(t)>\alpha_{\mathrm{R}}^{\prime}, \quad \text { for } x=0,
$$

for $|t|$ smaller than its value at the dip. Meanwhile

$$
\alpha_{\text {eff }}^{\prime}(t)<\alpha_{\mathrm{R}}^{\prime}, \quad \text { for } x=2
$$

\subsection{Comparison with experiment}

We will now discuss comparison with experiment and the uncertainties of the present theory. Two typical experiments will be considered: one with particularly large $\alpha_{\text {eff }} \pi \mathrm{N}$ charge exchange, and one with small $\alpha_{\text {eff }}$, charged $\pi$ photoproduction.

There is some evidence in $\pi \mathrm{N}$ charge exchange of the kink at the dip although it is not statistically significant $[57,58]$. See fig. 8. However, measurements beyond the dip are not accurate and the theory is not expected to be good on this detail at low energies because of double Reggeon exchange contributions and perhaps $t$-channel unitarity effects. There is stronger evidence for this kink in $\gamma p \rightarrow \pi^{0} p$ (ref. [56]), fig. 9. (High energy $\gamma p \rightarrow \pi^{\circ} p$ at smaller angle should show $\alpha_{\text {eff }} \approx \alpha_{\rho}$ pole.)

Aside from the detailed kink structure, the whole secondary maximum region in $\pi \mathrm{N}$ charge exchange has an experimental $\alpha_{\text {eff }}$ considerably smaller than theory, if $\alpha_{V}^{+}=0$ (non-shrinking diffractive scattering). It is quite possible to reduce $\alpha_{\text {eff }}$ so that it roughly agrees with the experimental $\alpha_{\text {eff }}$ if $\alpha \dot{\mathrm{V}} \approx 1$. However, that would cause trouble with charged $\pi$-photoproduction discussed below. We prefer to argue that double Reggeon exchange contributions (or lower trajectory or background contributions) are important at the lower energies or $t$-channel unitarity corrections to the $n=0$ amplitude are important. (These would reduce $\alpha_{\text {eff }}$ of the large $n=0$ cut in the region

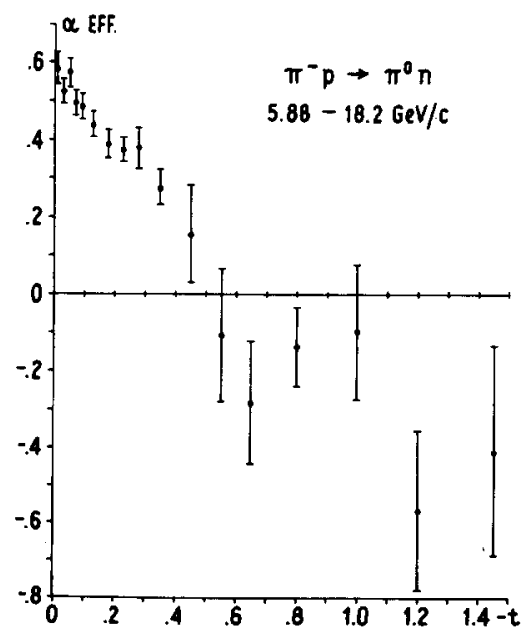

Fig. 8. $\alpha_{\text {eff }}$ for pi-nucleon charge exchange. A kink in $\alpha_{\text {eff }}$ at $-t \approx 0.6$, as predicted by SCRAM, is visible, although not statistically significant. 


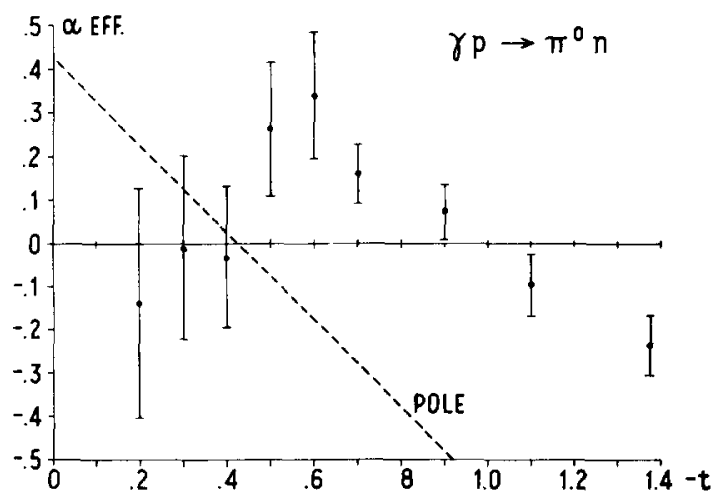

Fig. 9. $\alpha_{\text {eff for }} \pi^{0}$ photoproduction. The kink and the higher $\alpha_{\text {eff }}$, as predicted by SCRAM, are clearly visible.

of the secondary maximum as discussed below.) Some evidence for the former is obtained by examining $\alpha_{\text {eff }}(t)$ calculated from data in the 4.6-18 and $5.9-18 \mathrm{GeV} / c$ regions. The theory is insensitive to this small change in energy interval. The experimental $\alpha_{\text {eff }}$ rises significantly as the minimum energy is increased.

Charged $\pi$-photoproduction is largely evasive $\pi$-exchange at small $-t$, going over to large contributions from $\rho, A_{2}$ at larger $-t$. The net $\alpha_{\text {eff }}(t)$ should be rather constant near $\alpha=0$ for $-t \lesssim 1$ if $\alpha_{\mathrm{V}}^{\prime} \approx 0$. At first the $\pi$-cut dominates, then $\rho, \mathrm{A}$-pole, then $\rho, \mathrm{A}$-cut. Qualitatively this prediction is just what is observed (fig. 10) (ref. [621). The prediction would be less satisfactory if $\alpha_{V}^{\prime} \approx 1$; then we expect $\alpha_{\text {eff }}^{\prime} \approx \frac{1}{2}$ beyond $-t \approx-t \approx 0.2$, which would be too large for experiment.

We conclude that if $\alpha_{\mathrm{V}}^{\prime} \ll 1$, i.e., diffractive scattering is not shrinking rapidly, then the strong cut model predicts roughly constant $\alpha_{\text {eff }} \approx 0$ differential cross sections for processes with $\pi$-exchange in agreement with the remarkable experimental results. However, with the same model it is difficult to obtain a large enough $\alpha_{\text {eff }}^{\prime}$ to agree with experiments on $\pi \mathrm{N}$ charge exchange.

It has recently been argued by Fox [80] that this relatively poor fit to the larger angle data $(-t \approx 1)$ for $\pi^{-} \mathrm{p} \rightarrow \pi^{\circ} \mathrm{n}$ is a significant difficulty for a model such as ours. Such a view is, however, rather naive, because it is in the same angular region that known approximations are expected to fall, as mentioned above. For example, double-particle exchange effects are important and are known to go in the direction of improving agreement between experiment and theory. For example, if, instead of the "asymptotic" or diffractive part of the elastic scattering, one used the observed elastic scattering (presumably including particle exchange) in an absorption calculation the motion of pole-cut interference dips is to larger rather than smaller $-t$ in the $5 \mathrm{GeV} / c$ region, eliminating the kink of fig. 6 , and $\alpha_{\text {eff }}$ is significantly reduced. In addition, $t$-channel unitarity, discussed below, implies that the cut discontinuities must vanish at the branch point in the angular momentum plane [82]. It can be shown that this constraint is in fact satisfied for all 


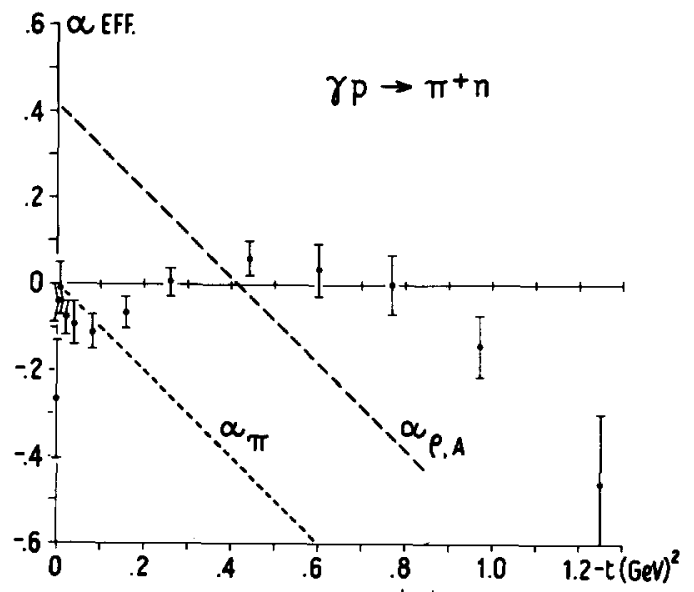

Fig. 10. $\alpha_{\text {eff for }} \pi^{+}$photoproduction. At small $|t|$ pion exchange dominates; at larger $|t|$ the $\rho, A_{2}$ and their associated cuts dominate.

photoproduction amplitudes in our model and the $n=1$ amplitude in $\pi^{-} \mathrm{p} \rightarrow \pi^{\circ} \mathrm{n}$, while it is violated for the $n=0$ amplitude in $\pi^{-} \mathrm{p} \rightarrow \pi^{\mathrm{O}} \mathrm{n}$. Thus the latter can be expected to receive significant modifications compared to the photoproduction amplitudes or the $n=1$ amplitude in $\pi^{-} \mathrm{p} \rightarrow \pi^{\mathrm{o}} \mathrm{n}$. For $-t \approx 0.6$ in $\pi^{-} \mathrm{p} \rightarrow \pi^{\circ} \mathrm{n}$ where the $n=0$ amplitude is important, we consequently expect theory to show some disagreement with data, while the agreement between experiment and theory should be better for $\gamma \mathrm{p} \rightarrow \pi^{\circ} \mathrm{p}$. The data indeed is as we expect.

\subsection{Energy dependence of the cut, t-channel unitarity}

In this section we assume that $\alpha_{\mathrm{V}}=1$, i.e., that $T$ eff $(b)$ has no $s$-dependence. Then from eq. (29) we have

$$
\begin{aligned}
M^{\mathrm{C}} & \approx \frac{1}{2} s \int b \mathrm{~d} b T^{\mathrm{eff}}(b) A^{P}(b) J(b \sqrt{-t}) \\
& =\frac{1}{2} \int b \mathrm{~d} b T^{\operatorname{eff}}(b) J_{n}(b \sqrt{-t}) \int \mathrm{d} t^{\prime} J_{n}\left(b \sqrt{-t^{\prime}}\right) M^{P}\left(t^{\prime}\right) .
\end{aligned}
$$

$M^{P}\left(t^{\dagger}\right)$ is given by eq. (27):

$$
M^{P}\left(t^{\prime}\right)=\left(-t^{\prime}\right)^{\frac{1}{2}(n+x)}\left(\frac{s}{s_{\mathrm{o}}}\right)^{\alpha_{0}+\alpha^{\prime} t^{\prime}} F_{1}\left(t^{\prime}\right),
$$

where $F_{1}\left(t^{\prime}\right)$ is a function which is finite at $t^{\prime}=0$, and can be expanded in a power series

$$
\sum_{j=0}^{\infty} C_{j}\left(t^{\prime}\right)^{j} .
$$

Similarly $F_{2}, \ldots, F_{5}$, used below are functions which are finite when their arguments vanish, and can be expanded in a power series. Thus the impor- 
tant behaviour is explicitly exhibited. At high energies we are most interested in the highest power of $s$, which is obtained for $t^{\prime} \approx 0$. Thus, the very high energy behaviour is found by expanding $J_{n}\left(b \sqrt{-t^{\prime}}\right)=\left(-t^{\dagger}\right)^{\frac{1}{2} n} F_{2}\left(b, t^{\prime}\right)$.

We combine this result with eqs. (44) and (45), and carry out the $b$-integral. Defining $y=n+\frac{1}{2} x$, we get

$$
\begin{aligned}
M^{\mathrm{C}} & =\int_{-\infty}^{\mathrm{o}} \mathrm{d} t^{\prime}\left(-t^{\prime}\right)^{y}\left(\frac{s}{s_{\mathrm{o}}}\right)^{\alpha_{\mathrm{O}}+\alpha^{\prime} t^{\prime}} F_{3}\left(t^{\prime}, t\right), \\
& =\int_{-\infty}^{\alpha_{0}} \mathrm{~d} \alpha\left(\alpha_{0}-\alpha\right)^{y}\left(\frac{s}{s_{\mathrm{o}}}\right)^{\alpha} F_{4}(\alpha, t),
\end{aligned}
$$

where the substitution $t^{\prime}=\left(\alpha-\alpha_{0}\right) / \alpha^{\prime}$ has been made. Carrying out the $\alpha$-integration by the replacement $x=\left(\alpha_{0}-\alpha\right) \ln \left(s / s_{0}\right)$, we get

$$
M^{\mathrm{C}}=\left(\frac{s}{s_{\mathrm{o}}}\right)^{\alpha_{0}} \frac{1}{\left(\ln \left(s / s_{\mathrm{O}}\right)\right)^{y+1}} F_{5}\left(\frac{1}{\ln \left(s / s_{\mathrm{O}}\right)}, t\right),
$$

where, as mentioned above, $F_{5}$ can be expanded in a series of non-negative powers of $1 / \ln s$.

The $J$-plane singularity given by eq. (46) is a cut with a branch point at $J=\alpha_{0}$. The discontinuity is proportional to the coefficient of $s^{\alpha}$ in (46) and thus contains a factor $\left(\alpha_{0}-\alpha\right)^{n+\frac{1}{2} x}$, vanishing at the branch point in the $J$ plane if $n+\frac{1}{2} x>0$. Therefore our cut obeys the Bronzan-Jones constraint [82] ${ }^{*}$, except for $n=x=0$. In principle, iteration of processes in the $t$ channel ( $t$-channel unitarity) also leads to a vanishing of the discontinuity at the branch point for $n=x=0$. Thus, phenomenological effects of $t$-channel unitarity can probably be safely neglected except in this latter case.

\subsection{Duality and exchange degeneracy}

In the conventional Regge-pole models, a consistent set of assumptions have been made. These assumptions include "duality", exchange degeneracy, and the existence of wrong-signature zeros at negative $t$. It is widely believed that acceptance of any one of these assumptions precludes the possibility of strong cuts. We show, in this section, that this belief is incorrect, and in particular that slightly modified duality and exchange degeneracy are consistent with strong cuts.

We first review the situation in the conventional models. "Duality" [83, 84] is the assumption that the imaginary part of the asymptotic amplitude (Regge poles, in this case) is related to the low-energy resonance parameters in such a way that when resonances exist the imaginary part of the high-energy amplitude is large, and when there are no resonances it is small. When one applies this assumption to exotic quantum number processes, i.e., those without resonances, one concludes that the contributing Regge poles must

\footnotetext{
* The discontinuity should actually be singular at the branch point. Thus the above result is not exact.
} 
occur in pairs, one of each signature, with roughly the same trajectory, with the residue functions related in such a way as to make the imaginary part small. This pairing is known as exchange degeneracy. As an example, the $\rho$ and $A_{2}$ are supposed to be degenerate.

Another related assumption is the existence of wrong-signature zeros at negative $t$. We continue the example of the $\rho$ and $A_{2}$. At $\alpha=0$, the $t$-value of the $\mathrm{A}_{2}$ is negative. As a result, the $\mathrm{A}_{2}$ trajectory contains no spin-zero particle, and, since the signature phase $e^{-\frac{1}{2} i \pi \alpha}$ is purely real at $\alpha=0$, the imaginary part of the $A_{2}$ contribution vanishes there. Since the $\rho$-contribution is purely imaginary at $\alpha=0$, if the exchange degeneracy assumption is adopted, the $\rho$-contribution vanishes at $\alpha=0$, leading to a zero in $\rho$-dominated amplitudes [34, 35].

All these assumptions can be related to one more, namely that third double spectral function (3DSF) effects are weak. This is best understood by comparison with the opposite case, in which 3DSF's are important. It should be noted that there is no independent justification for this assumptions; a priori, one would expect the effects of all three double spectral functions to be comparable in size.

In SCRAM, 3DSF effects turn out to be large. In the first place, the absorption correction is a Regge cut, which requires the presence of a 3DSF [85]. Thus a strong cut is equivalent to a large 3DSF effect. Also, fixed poles are 3DSF effects.

SCRAM assumes that the Regge pole contributions have no zeros as a function of $t$. This requires that their residues in the angular momentum plane have poles at nonsense wrong-signature points. These poles presumably arise by mixing with Gribov-Pomeranchuk fixed poles [37-39]. These poles are caused by the 3DSF; and cuts, also 3DSF effects, are required to prevent the Gribov-Pomeranchuk pole from becoming an essential singularity. Thus this aspect of SCRAM, namely that it has no wrong-signature zeros, also is equivalent to large 3DSF effects*.

An appropriate duality assumption can be made in the presence of strong cuts. The resonances must be related to the sum of poles and cuts, rather than to the poles alone. Thus, in those cases in which the imaginary part at low energies is small, there can be a large imaginary part in the pole contribution if it is cancelled by the cut contribution.

A quantitative formulation of duality is provided by the finite energy sum rules [87]. These sum rules can distinguish the approximate location and strength of singularities in the angular momentum plane, but cannot distinguish between a pole and a cut. This was explicitly shown in a calculation by Jackson and Quigg $[88,45]$. They showed that the finite-energy sum rule worked almost equally well for pole models and for pole + cut models. They found that, the better the high-energy fit to the data was, the better the sum rule worked, independently of the nature of the $j$-plane singularity.

The modification needed in duality in the presence of strong cuts leads to a modification of exchange degeneracy. In order for duality to work for

\footnotetext{
* The large strength of the Gribov-Pomeranchuk fixed pole is determined using the Schwarz sum rules [86].
} 
exotic quantum number processes, the $\alpha$-values for the relevant trajectories must still be close together, at least at small $-t$, but the residues are not related in the same way as in the pure pole case, since cuts must be included. In particular, there is no condition that the residue of a Regge pole must vanish at wrong-signature points. The "simplicity mechanism" [32] is consistent with duality and exchange degeneracy in the presence of strong cuts.

Thus, the assumptions of duality and exchange degeneracy can be made in a consistent fashion either in a pure Regge pole model or in a model with strong cuts. This consistency is extended by the importance of third double spectral function effects, which are unimportant in the pure pole models, and important in SCRAM. The consistency, of course, does not establish that the duality assumption is correct. It is equally consistent to not have duality in either of the models. In any case, however, duality has nothing direct to say on the choice between the conventional models and SCRAM.

We remarked above that the conventional model $\mathrm{V}$, suggested by exchange degeneracy, was the closest to SCRAM, in that it had one zero per amplitude for vector meson exchange, although the positions of the zeros were incorrect in the V-model. The connection between V and SCRAM might be related to duality. It is not possible at present, however, to make any firm statements on the connection.

We would like to thank Nigel Buttimore, Geoffrey Fox, Robert Kelly, David Richards and Roy Schult for helpful comments.

\section{REFERENCES}

[1] F. Henyey, G. L. Kane, J. Pumplin and M. Ross, Phys. Rev. Letters 21 (1968) 946; Phys. Rev. 182 (1969) 1579, referred to as HKPR.

[2] V. Barger, Rev. Mod. Phys. 40 (1968) 129.

[3] G. Höhler et al., Phys. Letters 20 (1966) 79.

[4] B. Richter, Proc. of the 14th Int. Conf. on high energy physics, Vienna 1968.

[5] C. B. Chiu and J.D. Stack, Phys. Rev. 153 (1967) 1575.

[6] V. Barger and D. Cline, Phenomenological theories of high energy scattering (Benjamin, 1969).

[7] B. C. Barish et al., Phys. Rev. Letters 23 (1967) 607, and references therein.

[8] $\pi^{-} \mathrm{p} \rightarrow \mathrm{p} \pi^{-}:$S.W. Kormanyos et al., Phys. Rev. 164 (1967) 1661.

[9] $\pi^{+} p \rightarrow p \pi^{+}:$T. Dobrowolski et al., Phys. Letters 24B (1967) 203.

[10] $\mathrm{pp} \rightarrow \pi^{-} \pi^{+}$: C. T. Murphy, private communication.

[11] B. M. Udgaonkar and M. Gell-Mann, Phys. Rev. Letters 8 (1962) 346.

[12] J. Banaigs et al., Nucl. Phys. B9 (1969) 640.

[13] H. Nicholson et al., Phys. Rev. Letters 23 (1969) 603.

[14] M. Abolins et al., Phys. Rev. Letters 22 (1969) 427.

[15] H.W. Atherton et al., Phys. Letters 30B (1969) 494.

[16] J.S. Loos, U.E. Kruse and E. L. Goldwasser, Phys. Rev. 173 (1968) 1330.

[17] O.I. Dahl et al., Phys. Rev. 163 (1967) 1430; D. Meyer, private communication.

[18] A. M. Boyarski et al., reported by K. Lubelsmeyer, Proc. of 4th Int. Symp. on electron and photon interactions at high energies, ed. D. W. Braben, Daresbury Nuclear Physics Laboratory (1969). 
[19] C. Michael, Phys. Letters 29B (1969) 230.

[20] F. Henyey, A note on the phase problem in Proc. of the 1969 Regge-cut Conf. (Madison, Wisconsin (1969)).

[21] N. J. Sopkovich, Nuovo Cimento 26 (1962) 186.

[22] J.D. Jackson, Peripheral interactions in high-energy physics, Les Houches, 1965 , ed. C. DeWitt and M. Jacob (Gordon and Breach, 1966).

[23] K. Gottfried and J. D. Jackson, Nuovo Cimento 33 (1964) 309; 34 (1964) 735.

[24] L. Durand, III and Y. T. Chiu, Phys. Rev. 139 (1965) B646.

[25a] R. C. Arnold, Phys. Rev. 153 (1967) 1523.

[25b] G. Cohen-Tannoudji, A.Morel and H.Navelet, Nuovo Cimento 48A (1967) 1075.

[25c] V. Gribov, JETP 26 (1963) 414.

[26] D. G. Ravenhall and H.W. Wyld, Phys. Rev. Letters 21 (1968) 1770.

[27] M. Ross, in Proc. of the Boulder Conf., ed. K. T. Mahanthappa (Colorado University, 1969).

[28] G. Cohen-Tannoudji, private communication.

[29] R. Kelly, G. L. Kane, F. Henyey, Phys. Rev. Letters 24 (1970) 1511.

[30] G. L. Kane, F. Henyey, D. Richards, M. Ross and G. Williamson, to be published.

[31] L. Bertocchi, in Proc. of the Heidelberg Conf. on elementary particles (NorthHolland and Wiley, 1967).

[32] F. Henyey and G. L. Kane, Phys. Rev. 180 (1969) 1607.

[33] G. E. Hite, Rev. Mod. Phys. 41 (1969) 669.

[34] J. Finkelstein, Phys. Rev. Letters 22 (1969) 362.

[35] R. Arnold, Phys. Rev. Letters 14 (1965) 657.

[36] G. Veneziano, Nuovo Cimento 57 (1968) 190.

[37] A. H. Mueller and T. L. Trueman, Phys. Rev. 160 (1967) 1296.

[38] S. Mandelstam and L. L. Wang, Phys. Rev. 160 (1967) 1490.

[39] C. E. Jones and V. L. Teplitz, Phys. Rev. 159 (1967) 1271.

[40] G. Cohen-Tannoudji, A.Morel and H. Navelet, Nuovo Cimento 48A (1967) 1075; G. Cohen-Tannoudji, P.Salan and A.Morel, Nuovo Cimento 55A (1961) 412, footnote p. 421.

[41] B.J.Hartley, R.W.Moore and K.J.M.Moriarty, Phys. Rev. 187 (1969) 339.

[42] P. A. Collins et al., unpublished.

[43] A.Dar and V. Weisskopf, Proc. of McGill University Summer School, 1969, ed. B. Margolis;

A. Dar, T. Watts and V. Weisskopf, Nucl. Phys. B13 (1969) 477.

[44] R. Carlitz and M. Kislinger, Calif. Inst. of Tech. preprint (1970).

[45] J. D. Jackson, Rev. Mod. Phys. 42 (1970) 12 and references contained therein.

[46] R. C. Arnold, Regge-cuts in elastic scattering, in the Proc. of the 1969 Reggecut Conf., ed. P.M. Fishbane and L.M.Simmons (Univ, of Wisconsin, Madison); and private communications.

[47] M. L. Blackmon and G. R. Goldstein, Phys. Rev. 179 (1969) 1480, and references contained therein.

[48] K. J. Foley et al., Phys. Rev. Letters 10 (1963) 376; 11 (1963) 425, 503;

15 (1965) 45;

J. Banaigs et al., ref. [12];

ABCLV Collaboration, Phys. Rev. Letters 23 (1969) 192.

[49] V. Barger and L. Durand, III, Phys. Rev. Letters 19 (1967) 1295.

[50] W. F. Baker et al., Phys. Letters 28B (1968) 291.

[51] D. P. Owen et al., Phys. Rev. 181 (1969) 1794.

[52] D. J. Crennell et al., Phys. Rev. Letters 23 (1969) 1347.

[53] R. Odorico, A simple phenomenological regularity of near-forward zeros, paper submitted to the Irvine conference on Regge poles, 1969.

[54] R.J. N. Phillips and W. Rarita, Phys. Rev. 139 (1965) B1336.

[55] H. Harari, in Proc. of 4th Int. Symp. on electron and photon interactions at high energies, ed. D.W. Braben (Daresbury Nuclear Physics Laboratory, 1969).

[56] $\gamma p \rightarrow \pi^{0} p, \gamma p \rightarrow \eta p:$ R. Anderson et al., Phys. Rev. Letters 21 (1968) 384 and SLAC preprint, Aug. 1969. 
[57] $\pi^{-} p \rightarrow \pi^{\circ} \mathrm{n}$ : A. V. Stirling et al., Phys. Rev. Letters 14 (1965) 763.

[58] P. Sonderegger et al., Phys. Letters 20 (1966) 75.

[59] $\pi_{p}^{+} p \rightarrow \pi^{\circ} \Delta^{+F}:$ G. Gidal et al., Phys. Rev. Letters 23 (1969) 994.

[60] $\pi^{+} n \rightarrow \omega p:$ G. C. Benson et al., Phys. Rev. Letters 22 (1969) 1074; G.S. Abrams et al., Phys. Rev. Letters 23 (1969) 673.

[61] $\pi^{+} p \rightarrow \omega \Delta^{++}$: Aachen-Berlin-CERN Collaboration, Nucl. Phys. B8 (1968) 485; B8 (1968) 45 .

[62] $\gamma p \rightarrow \pi^{+} n, \gamma n \rightarrow \pi^{-} p:$ A. M. Boyarski et al., Phys. Rev. Letters 20 (1968) 300 and 21 (1968) 1767;

P. Heide et al., Phys. Rev. Letters 21 (1968) 248.

[63] np $\rightarrow$ pn: G. Manning et al., Nuovo Cimento 41 (1966) 167;

E. L.Miller, M. Elfield, N.W. Reay, N.R.Stanton, M. A. Abolins, M. T. Lin, K. W. Edwards, Bull Am. Phys. Soc. Series II, 15 (1970) 660.

[64] $\mathrm{p} \overline{\mathrm{p}} \rightarrow \mathrm{n} \overline{\mathrm{n}}:$ P. Astbury et al., Phys. Letters 22 (1966) 537 and 23 (1966) 160.

[65] M. LeBellac, Phys. Letters 25B (1967) 524.

[66a] J. Fr $\not y l$ and and D. Gordon, Phys. Rev. 177 (1969) 2500.

[66b] D. Amati et al., Phys. Letters 26B (1968) 510.

[66c] A. Kaidalov and S. Karnakhov, Phys. Letters 29B (1969) 372, 376;

Benfatto, Nicolo and Rossi, Nuovo Cim. Letters 1 (1969) 537 and private communication, and ref. [30].

[66d] N. Byers and G. H. Thomas, Phys. Rev. Letters 20 (1968) 129.

[67] I. Bender, H. G. Dosch and H.J. Rothe, Nuovo Cimento 62 (1969) 1026.

[68] B. Richter, High-energy photoproduction in the Proc. of the 1967 Int. Symp. on electron and photon interactions at high energies (SLAC, Sept., 1967).

[69] H. R. Rubinstein, G. Veneziano and A. M. Virasoro, Phys. Rev. 167 (1968) 1441.

[70] R. Dashen and S. Y. Lee, Phys. Rev. Letters 22 (1969) 366.

[71] $\mathrm{pp} \rightarrow \mathrm{n} \Delta^{++}:$Z. Ming Ma et al., Phys. Rev. Letters 24 (1970) 1031.

[72] J.H.Scharenguivel et al., Phys. Rev. Letters 24 (1970) 332, and references therein.

[73] F.S. Henyey, Phys. Rev. 170 (1968) 1619.

[74] V. Barger, C. Michael and R.J. N. Phillips, Phys. Rev. 185 (1969) 1852.

[75] B. Kayser, Phys. Rev. D1 (1970) 306.

[76] C. Chiu and M. Dersarkissian, Nuovo Cimento 55A (1968) 396.

[77] L. Sowash, private communication.

[78] G. L. Kane and M. Ross, Phys. Rev. 177 (1969) 2353;

H. Harari, ref. [55].

[79] M. L. Blackmon and G. R. Goldstein, Regge-pole eikonal theory of small-angle $\mathrm{K}_{2}^{\mathrm{O}}$ nucleon scattering, Syracuse preprint, Dec. 1969.

[80] G. Fox, in High energy collisions, Stony Brook 1969 (Gordon and Breach, New York, 1969).

[81] D. Amati, S. Fubini and A. Stenghellini, Phys. Letters 1 (1962) 29; Nuovo Cimento 26 (1962) 896.

[82] J. B. Bronzan and C. E. Jones, Phys. Rev. 160 (1967) 1494.

[83] G. Chew and A. Pignotti, Phys. Rev. Letters 20 (1968) 1078.

[84] C. Schmid, Phys. Rev. Letters 20 (1968) 689.

[85] S. Mandelstam, Nuovo Cimento 30 (1963) 1127, 1148.

[86] J. Schwarz, Phys. Rev. 159 (1967) 1269.

[87] R. Dolen, D. Horn and C. Schmid, Phys. Rev. 166 (1968) 1768.

[88] J.D. Jackson and C. Quigg, Phys. Letters 29B (1969) 236. 